\title{
ON-DIAGONAL LOWER BOUNDS FOR HEAT KERNELS AND MARKOV CHAINS
}

\author{
Thierry Coulhon \\ Université de Cergy-Pontoise \\ 95302 Cergy Pontoise Cedex \\ France \\ email: coulhon@u-cergy.fr \\ Alexander Grigor'yan \\ Imperial College \\ London SW7 2BZ, UK \\ email: a.grigoryan@ic.ac.uk
}

\section{Introduction}

Let $M$ be a Riemannian manifold, and $\Delta$ be the Laplace-Beltrami operator on $M$. It is known that there exists a unique minimal positive fundamental solution to the associated heat equation, which is referred to as the heat kernel and denoted by $p_{t}(x, y)(x, y \in M$, $t>0)$.

For example, in $\mathbb{R}^{n}$, the heat kernel is given by the explicit formula

$$
p_{t}(x, y)=\frac{1}{(4 \pi t)^{n / 2}} \exp \left(-\frac{|x-y|^{2}}{4 t}\right)
$$

which shows that $p_{t}(x, y)$ behaves like $t^{-\frac{n}{2}}$ for fixed $x$ and $y$ as $t \rightarrow \infty$. On other manifolds, its behaviour may be described by different functions of $t$, depending on the geometry of the manifold.

The major question on complete non-compact manifolds is: What geometric terms are adequate to describe the long time behaviour of the heat kernel. Much has been known about upper bounds. The seminal works of Nash [N], Aronson [Ar], Varopoulos [V2], Carlen, Kusuoka, Stroock [CKS] and Davies [D1] have brought the understanding that the uniform upper bounds of the heat kernel are closely related to isoperimetric type inequalities including the Sobolev's, Nash's and the logarithmic Sobolev inequalities. More recent works [G2], [Carr], [C2] revealed the importance of a Faber-Krahn type inequality and of a generalized Nash inequality (see also the surveys [G4] and [C3]).

The situation is quite different with lower bounds of the heat kernel. Until recently, only two methods were known:

- a comparison type theorem ([DGM], $[\mathrm{ChY}])$ which requires a pointwise restriction on the Ricci curvature;

Coulhon T., Grigor'yan A., On-diagonal lower bounds for heat kernels on noncompact manifolds and Markov chains, Duke Math. J., 89 (1997) 
- using an upper bound of the heat kernel, together with a uniform Harnack inequality or equivalent tools (see $[\mathrm{N}],[\mathrm{Ar}],[\mathrm{LY}],[\mathrm{FS}],[\mathrm{BCF}],[\mathrm{PE}]$ ).

The latter approach needs additional ingredients, essentially Poincaré type inequalities and the doubling volume property (see [S], [G6]). In particular, this means that it can only treat a polynomial decay of the heat kernel and that its natural range is limited to manifolds with a non-negative Ricci curvature (up to quasi-isometry). On the other hand, both above approaches give at the same time off-diagonal bounds for $p_{t}(x, y)$.

In the present paper, we propose an approach to the lower on-diagonal bounds for the heat kernel that is independent of the upper bounds and that works even when the Harnack principle is unknown or false. Therefore, the range of manifolds which can be treated by our method is much wider than before. A first step in that direction (mostly for a discrete time setting) appeared recently in the work of F.Lust-Piquard [L] but our results require fewer hypotheses and yield sharper estimates.

We also take the classical route that goes from upper bounds to lower bounds, but without assuming Poincaré inequalities, and we try to get endpoint results in that direction. Let us mention at once that, for the time being, we are unable to get off-diagonal lower bounds (anyway further assumptions are needed there).

We make alternatively two kinds of assumptions about the geometry of the manifold $M$ :

- an anti-isoperimetric (or anti-Faber-Krahn) type inequality;

- an upper bound of the volume growth function or a doubling volume property

The estimates we obtain are also of two kinds:

- a sup-lower bound i.e. a lower bound for $\sup _{x \in M} p_{t}(x, x)$;

- a pointwise lower bound i.e. a lower bound for $p_{t}(x, x)$ for a fixed given point $x \in M$.

As was shown by E.B.Davies [D2], these two functions may behave differently as $t \rightarrow \infty$.

Let $V(x, r)$ denote a volume of a geodesic ball of radius $r$ and centre $x \in M$. For any domain $\Omega \subset M$ we denote by $\lambda_{1}(\Omega)$ the bottom of the $L^{2}$-spectrum of $-\Delta$ on $\Omega$ with a Dirichlet boundary condition.

The structure of the paper is as follows.

In $\S 2$, we show that a sup-lower bound follows from a doubling volume property: if for some point $x_{0}$ and any $r>0$

$$
V\left(x_{0}, 2 r\right) \leq C V\left(x_{0}, r\right)
$$

then, for all $t>0$ and some $c>0$

$$
\sup _{x} p_{t}(x, x) \geq \frac{c}{V\left(x_{0}, \sqrt{t}\right)}
$$

Let us emphasize that the results of $\S 2$ and partially of $\S 3$ are obtained in the setting of a general semigroup theory.

In $\S 3$, we shall see that sup-lower bounds can be obtained from certain types of antiisoperimetric inequalities. Let us say that a manifold $M$ satisfies an anti-Faber-Krahn inequality if there is a family $\left\{\Omega_{\xi}\right\}_{\xi>0}$ of regions of $M$ such that the volume of $\Omega_{\xi}$ is equal to $\xi$ and

$$
\lambda_{1}\left(\Omega_{\xi}\right) \leq \Lambda(\xi)
$$


for some function $\Lambda$. Given an anti-Faber-Krahn inequality, we deduce a sup-lower bound

$$
\sup _{x} p_{t}(x, x) \geq f(t)
$$

where the function $f$ depends on the function $\Lambda$ (see Theorem 3.2). A similar statement is true if (1.1) is replaced by another kind of anti-isoperimetric inequality where the boundary area of a family of balls is bounded from above by a certain function of their volume.

In $\S 4$, we develop the same theory for random walks on graphs. In $\S 5$, we gather partial results for upper and lower bounds in the case of a positive spectral gap.

In $\S 6$, we prove pointwise lower bounds of $p_{t}(x, x)$ in terms of a volume upper bound and of the local geometry around $x$. In particular, we show that if for all $r>r_{0}$ and some $x \in M$

$$
V(x, r) \leq C r^{n}
$$

then for all $t>t_{0}$

$$
p_{t}(x, x) \geq \frac{c}{(t \log t)^{\frac{n}{2}}}
$$

If (1.2) is replaced by a more general inequality

$$
V(x, r) \leq v(r)
$$

with a virtually arbitrary function $v(r)$ then we still have a pointwise lower bound of $p_{t}(x, x)$ in terms of another function of $t$ which is expressed via $v(r)$ (see Theorem 6.1).

In $\S 7$, we prove that $(1.3)$ can be sharpened to

$$
p_{t}(x, x) \geq \frac{\text { const }}{t^{\frac{n}{2}}}
$$

provided $M$ satisfies the doubling volume property and one knows a priori an upper bound

$$
p_{t}(x, x) \leq \frac{\text { const }}{t^{\frac{n}{2}}} .
$$

In $\S 8$ and $\S 9$, we show on examples of rotationally invariant manifolds that our results are optimal, in particular, the logarithm in the denominator of (1.3) cannot be in general got rid of.

In $\S 10$, we give a sup-lower bound under hypotheses similar to those of $\S 6$, but with a weaker assumption on the local geometry.

\section{Lower bounds and $\lambda_{1}$}

Let $(X, \mu)$ be a $\sigma$-finite measured space, and $T_{t}$ a semigroup of symmetric contractions of $L^{2}(X, \mu)$. Denote by $-A$ the infinitesimal generator of $T_{t}$. One has the following inequality

$$
\exp \left(-2 \frac{(A f, f)}{\|f\|_{2}^{2}} t\right) \leq \frac{\left\|T_{t} f\right\|_{2}^{2}}{\|f\|_{2}^{2}}, \forall f \in \mathcal{D}(A), \forall t>0
$$


This inequality can be easily proved by spectral theory ([C2]). It is the cornerstone of the semigroup version $([\mathrm{C} 2])$ of a theorem by the second author that relates upper bounds for the heat kernel with Faber-Krahn type inequalities ([G2]). We will see that it gives a very easy approach to sup-lower bounds for the heat kernels, and more generally the kernels of symmetric Markov semigroups. In the case where $(X, \mu)$ is a Riemannian manifold $M$ equiped with its natural measure, and $T_{t}$ is the heat semigroup, i.e. the heat kernel on a Riemannian manifold, the technique of [G2] also applies.

Recall that if $T_{t}$ has a kernel $p_{t}$ (and this is the case as soon as $\left\|T_{t}\right\|_{1 \rightarrow \infty}<+\infty$ ), then $\left\|T_{t}\right\|_{1 \rightarrow \infty}=\sup _{x \in X} p_{t}(x, x)$.

In the sequel, $\sup _{x \in X} p_{t}(x, x)$ will simply mean $+\infty$ if $\left\|T_{t}\right\|_{1 \rightarrow \infty}=+\infty$.

Let $\mathcal{D}$ be a dense subset of $\mathcal{D}(A) \backslash\{0\}$ in $L^{2}$ that is also contained in $L^{1}$. One easily deduces from (2.1):

\subsection{Proposition.}

$$
\sup _{x \in X} p_{t}(x, x) \geq \sup _{f \in \mathcal{D}}\left\{\frac{\|f\|_{2}^{2}}{\|f\|_{1}^{2}} \exp \left(-\frac{(A f, f)}{\|f\|_{2}^{2}} t\right)\right\} .
$$

Proof: For $f \in \mathcal{D}$, multiplying (2.1) by $\frac{\|f\|_{2}^{2}}{\|f\|_{1}^{2}}$ gives

$$
\frac{\|f\|_{2}^{2}}{\|f\|_{1}^{2}} \exp \left(-2 \frac{(A f, f)}{\|f\|_{2}^{2}} t\right) \leq \frac{\left\|T_{t} f\right\|_{2}^{2}}{\|f\|_{1}^{2}} \leq\left\|T_{t}\right\|_{1 \rightarrow 2}^{2} .
$$

Now $\left\|T_{t}\right\|_{1 \rightarrow 2}^{2}=\left\|T_{t}^{*} T_{t}\right\|_{1 \rightarrow \infty}=\left\|T_{2 t}\right\|_{1 \rightarrow \infty}$, and the proposition is proved.

\subsection{Corollary.}

$$
\sup _{x \in X} p_{t}(x, x) \geq e^{-1} \sup \left\{\frac{\|f\|_{2}^{2}}{\|f\|_{1}^{2}} ; f \in \mathcal{D},(A f, f) \leq \frac{1}{t}\|f\|_{2}^{2}\right\} .
$$

Define

$$
\lambda_{1}(\Omega)=\inf _{f \in \mathcal{D}, \operatorname{supp} f \subset \Omega} \frac{(A f, f)}{\|f\|_{2}^{2}},
$$

where $\Omega$ is a measurable subset of $X$ with finite measure $|\Omega|=\mu(\Omega)$. One gets from Proposition 2.1

\subsection{Proposition.}

$$
\sup _{x \in X} p_{t}(x, x) \geq \sup _{\Omega}\left\{\frac{1}{|\Omega|} \exp \left(-\lambda_{1}(\Omega) t\right)\right\}
$$

Proof: Fix $\Omega$ as above. If $f \in \mathcal{D}$ is supported in $\Omega,\|f\|_{1} \leq|\Omega|^{1 / 2}\|f\|_{2}$, therefore

$$
\begin{aligned}
& \sup _{f \in \mathcal{D}}\left\{\frac{\|f\|_{2}^{2}}{\|f\|_{1}^{2}} \exp \left(-\frac{(A f, f)}{\|f\|_{2}^{2}} t\right)\right\} \geq \sup _{f \in \mathcal{D}, \operatorname{supp} f \subset \Omega}\left\{\frac{1}{|\Omega|} \exp \left(-\frac{(A f, f)}{\|f\|_{2}^{2}} t\right)\right\} \\
= & \frac{1}{|\Omega|} \exp \left(-\inf _{f \in \mathcal{D}, \operatorname{supp} f \subset \Omega} \frac{(A f, f)}{\|f\|_{2}^{2}} t\right)=\frac{1}{|\Omega|} \exp \left(-\lambda_{1}(\Omega) t\right) .
\end{aligned}
$$

It follows from Proposition 2.1 that

$$
\sup _{x \in X} p_{t}(x, x) \geq \frac{1}{|\Omega|} \exp \left(-\lambda_{1}(\Omega) t\right),
$$

and one takes the supremum over $\Omega$. 


\subsection{Corollary.}

$$
\sup _{x \in X} p_{t}(x, x) \geq e^{-1} \sup _{\Omega}\left\{\frac{1}{|\Omega|} ; \lambda_{1}(\Omega) \leq \frac{1}{t}\right\}
$$

In other terms, suppose that for some $t \in \mathbb{R}_{+}^{*}$, there exists $\Omega_{t}$ such that $\lambda_{1}\left(\Omega_{t}\right) \leq \frac{1}{t}$ and $\left|\Omega_{t}\right| \leq v$. Then $\sup _{x \in X} p_{t}(x, x) \geq 1 /$ ev .

Let us recall that an upper bound of $\sup _{x \in X} p_{t}(x, x)$ is equivalent to a Faber-Krahn inequality of the type $\Lambda(|\Omega|) \leq \lambda_{1}(\Omega)$, where $\Lambda$ is a decreasing function (see [G2], [C2]). In other terms, one gets an upper bound on the kernel if one knows that the $\lambda_{1}$ of a set cannot be small unless the set is sufficiently large. What the above corollary says is that a lower bound follows if there are sets with a relatively small $\lambda_{1}$ (the scale being given by the time one is interested in) but that are not too large. The latter condition can be called an anti-Faber-Krahn inequality. The version we have just presented is very primitive. We shall give an optimal version in terms of a lower bound on $\sup _{x \in X} p_{t}(x, x)$ as a function of $t$ in $\S 3$. However, Corollary 2.4 has the advantage that it can be used at a definite scale of time.

Suppose now that $X$ is a metric space. Then the general principle of Corollary 2.4 that a lower bound of $\sup _{x \in X} p_{t}(x, x)$ follows if one has an upper bound on the $\lambda_{1}$ of a family of sets whose volume is under control can be in particular applied to balls. Denote by $B(x, r)$ the ball of center $x \in X$ and radius $r>0$ for the metric on $X$.

2.5 Corollary. Suppose that there exists a one-to-one mapping $\varphi$ from $\mathbb{R}_{+}^{*}$ to itself such that for every $r \in \mathbb{R}_{+}^{*}$, there exists $x_{r} \in M$ such that

$$
\lambda_{1}\left(B\left(x_{r}, r\right)\right) \leq \frac{1}{\varphi(r)}
$$

Then

$$
\sup _{x \in X} p_{t}(x, x) \geq \frac{1}{e V\left(x_{\varphi^{-1}(t)}, \varphi^{-1}(t)\right)}, \forall t>0
$$

Examples: Take for $X$ the $n$-dimensional hyperbolic space $H_{n}$, with a sequence of arbitrarily large euclidean balls embedded in it whose radii do not grow too fast. Since for a euclidean ball

$$
\lambda_{1}(B(x, r)) \leq \frac{C}{r^{2}}
$$

then one shows easily using Corollary 2.5

$$
\sup _{x \in X} p_{t}(x, x) \geq c t^{-n / 2}, \forall t>0
$$

According to [HS], such a manifold being Cartan-Hadamard satisfies the euclidean isoperimetric inequality, therefore the above estimate is optimal. Analogously, one can easily build $n$-manifolds with arbitrary volume growth such that

$$
\sup _{x \in X} p_{t}(x, x) \geq c t^{-1 / 2}, \forall t>1
$$

just by inserting or gluing arbitrarily long tubes, i.e. products of $S^{n-1}$ by an interval. 
Corollary 2.5 is not optimal in general (see 3.3 below), but it is if for example $\varphi(r)=r^{2}$, and this will enable us to derive an optimal lower bound in the case where $X$ has the doubling volume property.

Assume that $T_{t}$ is connected to the distance $d$ on $X$, in the sense that there exists a gradient $\nabla$ on $X$ such that $|\nabla d| \leq 1$ and $(A f, f)=\|\nabla f\|_{2}^{2}$. The basic example is again the heat semigroup on a Riemannian manifold, but also any semigroup generated by a second order subelliptic operator on a manifold endowed with the associated distance. A general framework can be found in a series of papers by Sturm (see for example [Stu]).

2.6 Lemma. Suppose that for some $x_{0} \in X$, one has the doubling volume property $V\left(x_{0}, 2 r\right) \leq C V\left(x_{0}, r\right), \forall r>0$. Then

$$
\lambda_{1}\left(B\left(x_{0}, r\right)\right) \leq \frac{4 C}{r^{2}}, \forall r>0
$$

Proof: Fix $r>0$. Let $f=\left(r-d\left(x_{0}, .\right)\right)_{+}$, suitably regularised. Since $|\nabla f| \leq 1$ on $B\left(x_{0}, r\right)$ and zero elsewhere, $\|\nabla f\|_{2}^{2} \leq V\left(x_{0}, r\right)$. Now $f \geq r / 2$ on $B\left(x_{0}, r / 2\right)$, therefore $\|f\|_{2}^{2} \geq(r / 2)^{2} V\left(x_{0}, r / 2\right)$. It follows that

$$
\lambda_{1}\left(B\left(x_{0}, r\right)\right) \leq \frac{\|\nabla f\|_{2}^{2}}{\|f\|_{2}^{2}} \leq \frac{V\left(x_{0}, r\right)}{(r / 2)^{2} V\left(x_{0}, r / 2\right)} \leq \frac{4 C}{r^{2}} .
$$

Now one deduces easily from 2.3 and 2.6 the following

2.7 Theorem. Suppose that for some $x_{0} \in X$, one has the doubling volume property $V\left(x_{0}, 2 r\right) \leq C V\left(x_{0}, r\right), \forall r>0$. Then

$$
\sup _{x \in X} p_{t}(x, x) \geq \frac{e^{-4 C}}{V\left(x_{0}, \sqrt{t}\right)}, \forall t>0 .
$$

Let us compare this statement with the first assertion in [L], Theorem 13. Our doubling volume condition is much weaker, and we get a lower bound directly in terms of the volume function rather than in terms of a polynomial upper bound. Subsequently, if for example $V(x, r) \leq C r^{D} \log r$, for all $x \in X$ and large $r$, we get for large $t$

$$
\sup _{x \in X} p_{t}(x, x) \geq \frac{c}{\sqrt{t^{D} \log t}}
$$

instead of

$$
\sup _{x \in X} p_{t}(x, x) \geq \frac{c_{\varepsilon}}{\sqrt{t^{D+\varepsilon}}} .
$$

Remark: Since the above approach does not require $T_{t}$ to be Markov, or any information on the $L^{1}-L^{1}$ or $L^{\infty}-L^{\infty}$ norm of $T_{t}$, it might prove useful in the study of the heat kernel on differential forms. 


\section{Anti-Faber-Krahn and anti-isoperimetric inequalities}

Let us return to the general setting of operator semigroups: let $(X, \mu)$ be a $\sigma$-finite measured space, and $T_{t}$ a semigroup of symmetric contractions of $L^{2}(X, \mu)$, with infinitesimal generator $-A$ and kernel $p_{t}$ when it exists (see $\S 2$ ).

Recall the following result on upper bounds, that first appeared in the context of the heat kernel on a Riemannian manifold ([G2]), and was then generalised to the setting of Markov semi-groups ([C2]). We shall say that an increasing differentiable function $\gamma$ from $\mathbb{R}_{+}^{*}$ into itself satisfies condition $(D)$ if there exists $\alpha>0$ such that

$$
f(u) \geq \alpha f(t), \forall u \in[t, 2 t], \forall t>0,
$$

where $f(t)=\frac{\gamma^{\prime}(t)}{\gamma(t)}$. This condition does not affect the rate of increase of $\gamma(t)$ as $t \rightarrow+\infty$.

For example, such functions as $\gamma(t)=\log ^{a} t, t^{b}, \exp \left(t^{c}\right)$ all satisfy the condition (D), provided $a, b, c>0$. On the contrary, $\gamma(t)=1-\exp (-t)$ does not satisfy it.

3.1 Theorem. Let $\gamma$ be an increasing $C^{1}$ bijection of $\mathbb{R}_{+}^{*}$. Then

$$
\sup _{x \in X} p_{t}(x, x) \leq \frac{1}{\gamma(t)}, \forall t>0
$$

implies

$$
\lambda_{1}(\Omega) \geq \frac{\alpha}{2} \Lambda(|\Omega|)
$$

for every $\Omega$ with finite measure in $X$, where $\Lambda$ and $\gamma$ are related by $\gamma^{\prime}(t)=\gamma(t) \Lambda(\gamma(t))$ or

$$
t=\int_{0}^{\gamma(t)} \frac{d v}{v \Lambda(v)}
$$

provided the integral above converges. If $T_{t}$ is Markov and $\gamma$ satisfies $(D)$ then a converse holds: the inequality

$$
\lambda_{1}(\Omega) \geq \Lambda(|\Omega|)
$$

for every $\Omega$ with finite measure in $X$ implies

$$
\sup _{x \in X} p_{t}(x, x) \leq \frac{\text { const }_{\delta}}{\gamma(\delta t)}, \forall t>0, \forall \delta \in(0,1)
$$

We are now able to state a symmetric theorem about lower bounds.

3.2 Theorem. Let $\gamma$ and $\Lambda$ be as above. Suppose that $\gamma$ satisfies $(D)$ and that for every $\xi \in \mathbb{R}_{+}^{*}$, there exists $\Omega_{\xi}$ such that $\left|\Omega_{\xi}\right| \leq \xi$ and $\lambda_{1}\left(\Omega_{\xi}\right) \leq \Lambda(\xi)$. Then

$$
\sup _{x \in X} p_{t}(x, x) \geq \frac{1}{\gamma(C t)}, \forall t>0
$$

where $C=2 / \alpha, \alpha$ being the constant in condition $(D)$. 
Proof: From Proposition 2.3 one deduces

$$
\sup _{x \in X} p_{t}(x, x) \geq \sup _{\xi>0} \frac{\exp \left(-\lambda_{1}\left(\Omega_{\xi}\right) t\right)}{\left|\Omega_{\xi}\right|}
$$

therefore

$$
\sup _{x \in X} p_{t}(x, x) \geq \sup _{\xi>0} \frac{\exp (-\Lambda(\xi) t)}{\xi}=\sup _{\tau>0} \frac{\exp (-\Lambda(\gamma(\tau)) t)}{\gamma(\tau)}=\sup _{\tau>0} \frac{\exp \left(-\frac{\gamma^{\prime}(\tau)}{\gamma(\tau)} t\right)}{\gamma(\tau)},
$$

if one sets $\tau=\gamma^{-1}(\xi)$. In order to get the result, it suffices to find $\tau$ such that

$$
\frac{\exp \left(-\frac{\gamma^{\prime}(\tau)}{\gamma(\tau)} t\right)}{\gamma(\tau)} \geq \frac{1}{\gamma(C t)}
$$

i.e.

$$
\frac{\gamma(C t)}{\gamma(\tau)} \geq \exp \left(\frac{\gamma^{\prime}(\tau)}{\gamma(\tau)} t\right)
$$

or

$$
\log \gamma(C t)-\log \gamma(\tau) \geq \frac{\gamma^{\prime}(\tau)}{\gamma(\tau)} t
$$

Choose now $\tau=t / \alpha$. One has then

$$
\log \gamma(C t)-\log \gamma(\tau)=\log \gamma(2 \tau)-\log \gamma(\tau)=\tau \frac{\gamma^{\prime}(a \tau)}{\gamma(a \tau)}
$$

with $a \in] 1,2[$. By condition $(D)$,

$$
\frac{\gamma^{\prime}(a \tau)}{\gamma(a \tau)} \geq \alpha \frac{\gamma^{\prime}(\tau)}{\gamma(\tau)}
$$

hence

$$
\log \gamma(C t)-\log \gamma(\tau) \geq \alpha \tau \frac{\gamma^{\prime}(\tau)}{\gamma(\tau)}=\frac{\gamma^{\prime}(\tau)}{\gamma(\tau)} t
$$

The theorem is proved.

We can now state a more sophisticated version of Corollary 2.5.

3.3 Corollary. Let $B_{r}, r>0$, be a family of measurable subsets of $X$ and $\varphi$ a mapping from $\mathbb{R}_{+}^{*}$ to itself such that for every $r \in \mathbb{R}_{+}^{*}$,

$$
\lambda_{1}\left(B_{r}\right) \leq \frac{1}{\varphi(r)}
$$

Let $v$ be a one-to-one mapping from $\mathbb{R}_{+}^{*}$ to itself such that

$$
\left|B_{r}\right| \leq v(r), \forall r>0
$$


Suppose that $\int_{0} \varphi \circ v^{-1}(\xi) \frac{d \xi}{\xi}$ converges and that $\gamma$ defined by

$$
t=\int_{0}^{\gamma(t)} \varphi \circ v^{-1}(\xi) \frac{d \xi}{\xi}
$$

satisfies $(D)$. Then

$$
\sup _{x \in X} p_{t}(x, x) \geq \frac{1}{\gamma(C t)}, \forall t>0
$$

Proof: For $\xi>0$, set $\Omega_{\xi}=B_{v^{-1}(\xi)}$. One has

$$
\left|\Omega_{\xi}\right| \leq \xi \text { and } \lambda_{1}\left(\Omega_{\xi}\right) \leq \frac{1}{\varphi \circ v^{-1}(\xi)},
$$

therefore one can apply 3.2 with $\Lambda=\frac{1}{\varphi \circ v^{-1}}$, whence the result.

\section{Remarks:}

1. The above estimate is better than 2.5 if

$$
\gamma(t) \ll v\left(\varphi^{-1}(t)\right)
$$

which is the case if

$$
t \ll \int_{0}^{v\left(\varphi^{-1}(t)\right)} \varphi \circ v^{-1}(\xi) \frac{d \xi}{\xi} .
$$

This happens for example if $\varphi \circ v^{-1}$ grows faster than any power function.

2. When $X$ is a metric space, a possible choice for $B_{r}$ is the family of balls $B\left(x_{0}, r\right)$, $x_{0} \in X$, since it is natural to assume that one controls their volume. On the other hand, there is no reason why their $\lambda_{1}$ should be in general particularly small with respect to their volume.

From now on, for the sake of simplicity, we will suppose that $X=M$ is a Riemannian $n$-manifold endowed with its Riemannian measure $\mu$ and that $T_{t}$ is the heat semigroup on $M$, but it is clear that the ideas below can be extended to more general geometries (e.g. a Lie group endowed with Hörmander vector fields or more generally a differentiable manifold endowed with a symmetric second-order subelliptic operator). Denote by $S(x, r)$ the $n-1$ dimensional Hausdorff measure of the sphere $\partial B(x, r)$.

3.4 Proposition. Fix $x_{0}$ in $M$. Suppose that $S\left(x_{0}, r\right)$ is non-decreasing as a function of $r$. Then

$$
\lambda_{1}\left(B\left(x_{0}, r\right)\right) \leq 4\left(\frac{S\left(x_{0}, r\right)}{V\left(x_{0}, r\right)}\right)^{2} .
$$

Proof: Fix $r>0$. We want to find a function $f$ supported in $B\left(x_{0}, r\right)$ such that

$$
\frac{\|\nabla f\|_{2}^{2}}{\|f\|_{2}^{2}} \leq 4\left(\frac{S\left(x_{0}, r\right)}{V\left(x_{0}, r\right)}\right)^{2} .
$$


Let $\left.r_{1} \in\right] 0, r\left[\right.$ to be chosen later, and $f(y)=g\left(d\left(x_{0}, y\right)\right)$, where

$$
g(s)=\inf \left(1,\left(\frac{r-s}{r_{1}}\right)_{+}\right)
$$

(of course $g$ should be regularised, but for simplification we ignore this in the calculations below).

Set $S\left(x_{0}, s\right)=\varphi(s)$. One has

$$
\frac{\|\nabla f\|_{2}^{2}}{\|f\|_{2}^{2}}=\frac{\int_{0}^{r} g^{\prime}(s)^{2} \varphi(s) d s}{\int_{0}^{r} g(s)^{2} \varphi(s) d s} .
$$

Set $A=\int_{0}^{r} g^{\prime}(s)^{2} \varphi(s) d s$ and $B=\int_{0}^{r} g(s)^{2} \varphi(s) d s$. Then

$$
A=\frac{1}{r_{1}^{2}} \int_{r-r_{1}}^{r} \varphi(s) d s \leq \frac{1}{r_{1}} \varphi(r)
$$

and

$$
B \geq \int_{0}^{r-r_{1}} \varphi(s) d s
$$

Therefore

$$
\frac{A}{B}=\frac{\|\nabla f\|_{2}^{2}}{\|f\|_{2}^{2}} \leq \frac{\varphi(r)}{r_{1} \int_{0}^{r-r_{1}} \varphi(s) d s}
$$

and the claim will be proved if we are able to choose $r_{1}$ such that

$$
\frac{\varphi(r)}{r_{1} \int_{0}^{r-r_{1}} \varphi(s) d s} \leq 4\left(\frac{S\left(x_{0}, r\right)}{V\left(x_{0}, r\right)}\right)^{2}=4 \frac{\varphi(r)^{2}}{\left(\int_{0}^{r} \varphi(s) d s\right)^{2}}
$$

in other terms

$$
\left(\int_{0}^{r} \varphi(s) d s\right)^{2} \leq 4 r_{1} \varphi(r) \int_{0}^{r-r_{1}} \varphi(s) d s
$$

Choose $r_{1}$ such that

$$
r_{1} \varphi(r)=\int_{0}^{r-r_{1}} \varphi(s) d s
$$

Then

$$
\left(\int_{0}^{r} \varphi(s) d s\right)^{2} \leq\left(\int_{0}^{r-r_{1}} \varphi(s) d s+r_{1} \varphi(r)\right)^{2}=4 r_{1} \varphi(r) \int_{0}^{r-r_{1}} \varphi(s) d s
$$

and we have what we wanted. 


\section{Remarks:}

1. If one does not assume $S\left(x_{0}, r\right)$ to be non-increasing, one gets

$$
\lambda_{1}\left(B\left(x_{0}, r\right)\right) \leq 4\left(\frac{\sup _{s \leq r} S\left(x_{0}, s\right)}{V\left(x_{0}, r\right)}\right)^{2} .
$$

In particular, if $S\left(x_{0}, r\right)$ is quasi-non-decreasing in $r$, i.e.

$$
S\left(x_{0}, r\right) \leq K S\left(x_{0}, s\right), r<s,
$$

one gets (3.1) with a constant $4 K^{2}$ instead of 4 . Inequality (3.1) cannot be obtained in general as follows from [CL].

2. Since $\lambda_{1}(B(x, r))$ is by definition non-increasing in $r$, one can in fact replace (3.1) by:

$$
\lambda_{1}(B(x, R)) \leq 4\left(\inf _{r \leq R} \frac{S(x, r)}{V(x, r)}\right)^{2} .
$$

We shall see in Lemma 8.2 below that this estimate is optimal (up to constants) in the case of rotationally invariant manifolds.

We shall now see that it follows from Theorem 3.2 and Proposition 3.4 that an antiisoperimetric inequality implies a lower bound on $\sup _{x \in M} p_{t}(x, x)$. One says that $M$ satisfies a $\varphi$-isoperimetric inequality, where $\varphi$ is a non-decreasing function, if

$$
\frac{|\Omega|}{\varphi(|\Omega|)} \leq C|\partial \Omega|
$$

for every compact domain $\Omega$ in $M$ with smooth boundary. Such an inequality implies the Faber-Krahn inequality

$$
\frac{1}{\varphi^{2}(|\Omega|)} \leq C^{\prime} \lambda_{1}(\Omega)
$$

(see [C3]). Let us say that $M$ satisfies a $\varphi$-anti-isoperimetric inequality if there exists $x_{0} \in M$ and $c>0$ such that $S\left(x_{0}, r\right)$ is quasi-non-decreasing with respect to $r$ and

$$
\frac{V\left(x_{0}, r\right)}{\varphi\left(V\left(x_{0}, r\right)\right)} \geq c S\left(x_{0}, r\right), \forall r>0
$$

3.5 Corollary. Suppose that $\gamma$ satisfies $(D)$, that $\varphi$ is non-decreasing and that they are related by $\gamma^{\prime}(t)=\frac{\gamma(t)}{\varphi^{2}(\gamma(t))}$ or $t=\int_{0}^{\gamma(t)} \frac{\varphi^{2}(v)}{v} d v$. If $M$ satisfies a $\varphi$-anti-isoperimetric inequality, then

$$
\sup _{x \in X} p_{t}(x, x) \geq \frac{1}{\gamma(C t)}, \forall t>0,
$$

where $C=\frac{8 K^{2}}{\alpha c^{2}}$.

Proof: By hypothesis

$$
\frac{S\left(x_{0}, r\right)}{V\left(x_{0}, r\right)} \leq \frac{c^{-1}}{\varphi\left(V\left(x_{0}, r\right)\right)},
$$

therefore by Proposition 3.4

$$
\lambda_{1}\left(B\left(x_{0}, r\right)\right) \leq \frac{4 K^{2} c^{-2}}{\varphi^{2}\left(V\left(x_{0}, r\right)\right)} .
$$

The result then follows from Theorem 3.2. 


\section{Examples:}

1. If there exists $x_{0} \in M$ and $C>0$ such that

$$
S\left(x_{0}, r\right) \leq C V\left(x_{0}, r\right)^{\frac{D-1}{D}}, \forall r>0
$$

then

$$
\sup _{x \in X} p_{t}(x, x) \geq c t^{-D / 2}, \forall t>0 .
$$

2. If there exists $x_{0} \in M$ and $c>0$ such that

$$
S\left(x_{0}, r\right) \leq C \frac{V\left(x_{0}, r\right)}{\left(\log V\left(x_{0}, r\right)\right)^{1 / \alpha}}, \forall r>r_{0}
$$

then

$$
\sup _{x \in X} p_{t}(x, x) \geq c \exp \left(-c t^{\frac{\alpha}{\alpha+2}}\right), \forall t>t_{0}
$$

The last example shows that it would be desirable to extend Lemma 3.4 to other families of sets than balls. Indeed there is no reason why a $\varphi$ isoperimetric inequality should be saturated by balls. For instance, on groups of exponential growth, one always has

$$
\frac{1}{\log |\Omega|} \leq C \frac{|\partial \Omega|}{|\Omega|},
$$

([CS1]). If the group is in addition polycyclic, there always exists a family of sets $\Omega_{n}$ and $c>0$ such that $\left|\Omega_{n}\right| \rightarrow+\infty$ and

$$
c \frac{\left|\partial \Omega_{n}\right|}{\left|\Omega_{n}\right|} \leq \frac{1}{\log \left|\Omega_{n}\right|}
$$

$([\mathrm{P}])$, but the $\Omega_{n}$ 's are far from being balls, because they grow in a non-isotropic way. Suppose however that one is able to show that for such sets

$$
\lambda_{1}\left(\Omega_{n}\right) \leq C^{\prime}\left(\frac{\left|\partial \Omega_{n}\right|}{\left|\Omega_{n}\right|}\right)^{2}
$$

This would yield

$$
\lambda_{1}\left(\Omega_{n}\right) \leq \frac{C^{\prime \prime}}{\left(\log \left|\Omega_{n}\right|\right)^{2}},
$$

therefore, by adapting Theorem 3.2, using the fact that $\Omega_{n}$ has at most exponential growth,

$$
p_{t}(e) \geq c e^{-C t^{1 / 3}}, \forall t>1
$$

This would give another approach to the optimal lower bound for heat kernels on polycyclic groups with exponential growth, that is due to Alexopoulos [Al]. We intend to come back to these matters in a future paper with Christophe Pittet. 


\section{Markov chains on graphs}

Let $T$ be a symmetric Markov operator acting on $L^{2}(X, \mu)$. Our starting point will be this time the inequality

$$
\left(\frac{\|T f\|_{2}^{2}}{\|f\|_{2}^{2}}\right)^{k} \leq \frac{\left\|T^{k} f\right\|_{2}^{2}}{\|f\|_{2}^{2}}, \forall f \in L^{2}, \forall k \in \mathbb{N}
$$

Again, this inequality can be proved by spectral theory, or even more directly (see [C2]). One gets as in $\S 2$

\subsection{Proposition.}

$$
\left\|T^{2 k}\right\|_{1 \rightarrow \infty} \geq \sup _{f \in L^{1} \cap L^{2}}\left\{\frac{\|f\|_{2}^{2}}{\|f\|_{1}^{2}}\left(\frac{\|T f\|_{2}^{2}}{\|f\|_{2}^{2}}\right)^{k}\right\} .
$$

For $\Omega$ a measurable subset of $X$ with finite measure $|\Omega|$, define

$$
a(\Omega)=\sup \left\{\frac{\|T f\|_{2}}{\|f\|_{2}} ; f \in L^{2}, \operatorname{supp} f \subset \Omega\right\}
$$

\subsection{Proposition.}

$$
\left\|T^{2 k}\right\|_{1 \rightarrow \infty} \geq \sup _{\Omega} \frac{a(\Omega)^{2 k}}{|\Omega|}
$$

In view of the applications, it is convenient to formulate the above inequalities in terms of the Dirichlet form $\mathcal{E}(f)=\|f\|_{2}^{2}-\|T f\|_{2}^{2}$. Since $\frac{\|f\|_{2}^{2}-\|T f\|_{2}^{2}}{\|T f\|_{2}^{2}} \geq \log \frac{\|f\|_{2}^{2}}{\|T f\|_{2}^{2}}$, one gets from Proposition 4.1

\subsection{Proposition.}

$$
\left\|T^{2 k}\right\|_{1 \rightarrow \infty} \geq \sup _{f \in L^{1} \cap L^{2}}\left\{\frac{\|f\|_{2}^{2}}{\|f\|_{1}^{2}} \exp \left(-\frac{\mathcal{E}(f)}{\|T f\|_{2}^{2}} k\right)\right\}
$$

Define now

$$
\lambda(\Omega)=\inf \left\{\frac{\mathcal{E}(f)}{\|T f\|_{2}^{2}} ; f \in L^{2}, \operatorname{supp} f \subset \Omega\right\} .
$$

One gets

\subsection{Proposition.}

$$
\left\|T^{2 k}\right\|_{1 \rightarrow \infty} \geq \sup _{\Omega}\left\{\frac{1}{|\Omega|} \exp (-\lambda(\Omega) k)\right\} .
$$


Take now for $X$ an infinite, connected graph. Write $x \sim y$ if $x, y \in X$ are neighbours or $x=y$. Let $n(x)-1$ be the number of neighbours of $x \in X$. Assume that $X$ is uniformly locally finite, i.e. $\sup _{x \in X} n(x)<+\infty$. We shall endow $X$ with the measure $\mu=n(x) d x$, that is equivalent to the counting measure, and with the natural graph metric $d$, i.e. $d(x, y)$ is the minimal number of steps necessary to join $x$ to $y$. For $x \in X$ and $n \in \mathbb{N}$, let $B(x, n)=\{y \in X ; d(x, y) \leq n\}$ and denote by $V(x, n)$ the cardinal of $B(x, n)$. If $f$ be a finitely supported function on $X$, define the length of its gradient by

$$
\left|\nabla_{X} f\right|(x)=|\nabla f|(x)=\sum_{y \in X, x \sim y}|f(x)-f(y)|
$$

Denote by $|\Omega|$ the cardinal of the set $\Omega \subset X$, and define $\partial \Omega=\{x \in \Omega ; \exists y \notin \Omega, y \sim x\}$.

Let $p$ be a Markov kernel on $X$, i.e. a function $p: X \times X \rightarrow \mathbb{R}_{+}$such that $\sum_{y \in X} p(x, y)=$ $1, \forall x \in X$. We shall say that $p$ is admissible if it is reversible with respect to $n$, i.e. $p(x, y) n(x)=p(y, x) n(y)$, if there exists $c>0$ satisfying $p(x, y) \geq c$ if $x, y \in X$ are neighbours or $x=y$, and if $p$ has bounded range, i.e. there exists $r_{0}$ such that $p(x, y)=0$ as soon as $d(x, y) \geq r_{0}$. Denote by $p_{k}$ the $k^{\text {th }}$ iterated kernel of $p: p_{1}=p$ and $p_{k}=$ $\sum_{z \in X} p_{k-1}(x, z) p(z, y)$. The standard kernel $q$, defined by $q(x, y)=\frac{1}{n(x)}$ if $y \sim x, 0$ otherwise, is admissible. If $p$ is admissible and $T$ is the operator defined by

$$
T f(x)=\sum_{y \in X} p(x, y) f(y)
$$

one checks easily that $T$ is symmetric Markovian on $\ell^{2}(X)$ and that

$$
C^{-1}\||\nabla f|\|_{2}^{2} \leq \mathcal{E}(f) \leq C\||\nabla f|\|_{2}^{2}
$$

From now on, $p$ will be an admissible kernel and $T$ the associated operator. In the above estimates we had to consider the quantity

$$
\lambda(\Omega)=\inf \left\{\frac{\mathcal{E}(f)}{\|T f\|_{2}^{2}} ; f \in L^{2}, \operatorname{supp} f \subset \Omega\right\}
$$

but the natural geometric quantity is rather

$$
\lambda_{1}(\Omega)=\inf \left\{\frac{\mathcal{E}(f)}{\|f\|_{2}^{2}} ; f \in L^{2}, \operatorname{supp} f \subset \Omega\right\} .
$$

which is uniformly comparable to

$$
\inf \left\{\frac{\|\nabla f\|_{2}^{2}}{\|f\|_{2}^{2}} ; f \in L^{2}, \operatorname{supp} f \subset \Omega\right\}
$$

However we have the 
4.5 Lemma. There exists $C>0$ such that

$$
\lambda_{1}(\Omega) \leq \lambda(\Omega) \leq C \lambda_{1}(\Omega)
$$

for every finite subset $\Omega$ of $X$.

Proof: The left-hand-side inequality is obvious. As for the other one, since $T$ is Markov, $\|T f\|_{2}^{2} \leq\|T|f|\|_{2}^{2}$, therefore

$$
\frac{\mathcal{E}(|f|)}{\|f\|_{2}^{2}} \leq \frac{\mathcal{E}(f)}{\|f\|_{2}^{2}},
$$

which means that in $\lambda_{1}(\Omega)$ one can restrict the infimum to nonnegative functions. Now, if $f \geq 0$,

$$
\|T f\|_{2}^{2}=\sum_{x \in X}\left(\sum_{y \in X} p(x, y) f(y)\right)^{2} \geq c \sum_{x \in X} f^{2}(x)=c\|f\|_{2}^{2}
$$

and

$$
\frac{\mathcal{E}(f)}{\|T f\|_{2}^{2}} \leq c^{-1} \frac{\mathcal{E}(f)}{\|f\|_{2}^{2}},
$$

which proves the claim by taking the infimum.

We refer to [C2], $\S \mathrm{IV}, \mathrm{V}$ for a characterisation of an upper bound on $\sup _{x \in X} p_{k}(x, x)=$ $\left\|T^{k}\right\|_{1 \rightarrow \infty}$ in terms of Faber-Krahn type inequalities and we pass to lower bounds. One proves as in 2.6

4.6 Theorem. Suppose that for some $x_{0} \in X$, one has the doubling volume property $V\left(x_{0}, 2 n\right) \leq C V\left(x_{0}, n\right), \forall n \in \mathbb{N}^{*}$. Then

$$
\sup _{x \in X} p_{k}(x, x) \geq \frac{C^{\prime}}{V\left(x_{0}, \sqrt{k}\right)}, \forall k \in \mathbb{N}^{*}
$$

Proof: One sees as in 2.6 that

$$
\lambda_{1}\left(B\left(x_{0}, n\right)\right) \leq \frac{C}{n^{2}},
$$

and the result for even $k$ is a consequence of 4.4 and 4.5 . Using $p(x, x) \geq c$, one easily derives a lower bound on $p_{k}(x, x)$ for every $k$.

The proof of the following statement is similar to the one of 3.2.

4.7 Theorem. Suppose that $\Lambda$ and $\gamma$ are as in 3.1, and that for every $\xi \in \mathbb{R}_{+}^{*}$, there exists $\Omega_{\xi}$ such that $\left|\Omega_{\xi}\right| \leq \xi$ and $\lambda_{1}\left(\Omega_{\xi}\right) \leq \Lambda(\xi)$. Then

$$
\sup _{x \in X} p_{k}(x, x) \geq \frac{1}{\gamma(C k)}, \forall k>0
$$

where $C>0$ depends on $\alpha$, the constant in condition $(D)$, on the constants of the graph $X$, and on the constants of the kernel $p$. 
One says that $X$ satisfies a $\varphi$-isoperimetric inequality if

$$
\frac{|\Omega|}{\varphi(|\Omega|)} \leq C|\partial \Omega|
$$

for every finite subset $\Omega$ of $X$. Again, this inequality implies

$$
\frac{1}{\varphi^{2}(|\Omega|)} \leq C^{\prime} \lambda_{1}(\Omega)
$$

(see [C3]). Let us say that $X$ satisfies a $\varphi$-anti-isoperimetric inequality, where $\varphi$ is a nondecreasing function, if there exist $x_{0} \in X$ and $K, c>0$ such that

$$
S\left(x_{0}, n\right) \leq K S\left(x_{0}, p\right), n>p
$$

and

$$
\frac{V\left(x_{0}, n\right)}{\varphi\left(V\left(x_{0}, n\right)\right)} \geq c S\left(x_{0}, n\right), \forall n \in \mathbb{N}^{*}
$$

One proves as in $\S 3$

4.8 Corollary. Suppose that $\gamma$ satisfies $(D)$, that $\varphi$ is non-decreasing and that they are related by $\gamma^{\prime}(t)=\frac{\gamma(t)}{\varphi^{2}(\gamma(t))}$ or $t=\int_{0}^{\gamma(t)} \frac{\varphi^{2}(v)}{v} d v$. If $X$ satisfies a $\varphi$-anti-isoperimetric inequality, then

$$
\sup _{x \in X} p_{k}(x, x) \geq \frac{1}{\gamma(C k)}, \forall k \in \mathbb{N}^{*}
$$

However, in our discrete setting, we can get a version of 3.4 for more general families of sets than balls, and therefore generalise 4.8.

4.9 Proposition Let $\Omega$ be a finite subset of $X$. Suppose that $\Omega=\cup_{i=1}^{n} \Omega_{i}$, where the $\Omega_{i}$ are disjoint, $\Omega_{n}=\partial \Omega$, and all neighbours of points in Omega $a_{i}$ are contained in $\Omega_{i-1} \cup \Omega_{i} \cup \Omega_{i+1}$. Then, if $\forall i=1, \ldots, n,\left|\Omega_{i}\right| \leq K|\partial \Omega|$,

$$
\lambda_{1}(\Omega) \leq C\left(\frac{|\partial \Omega|}{|\Omega|}\right)^{2},
$$

where $C$ only depends on the constants of the graph $X$ and $K$.

Remark: If one does not assume $\left|\Omega_{i}\right| \leq K\left|\Omega_{n}\right|$, one gets still

$$
\lambda_{1}(\Omega) \leq C\left(\frac{\max _{i}\left|\Omega_{i}\right|}{|\Omega|}\right)^{2} .
$$


Proof: Set $\alpha_{i}=\left|\Omega_{i}\right|$ and $\beta_{i}=\sum_{j=1}^{i} \alpha_{j}, i=1, \ldots, n$. We can assume for example $n \geq 3$ because otherwise $|\Omega|=\sum_{i=1}^{n}\left|\Omega_{i}\right| \leq(K+1)|\partial \Omega|$, therefore $\frac{|\partial \Omega|}{|\Omega|}$ is bounded below, which proves the claim since $\lambda_{1}(\Omega)$ is obviously bounded above.

Let us first treat the case where, say, $\beta_{n-1} \leq \alpha_{n}$, thus $\beta_{n} \leq 2 \alpha_{n}$. Take $f=1_{\Omega}$. Then

$$
\|f\|_{2}^{2}=\beta_{n}
$$

and

$$
\|\nabla f\|_{2}^{2}=\sum_{x \in \Omega}|\nabla f(x)|^{2}+\sum_{x \in X \backslash \Omega}|\nabla f(x)|^{2} \leq\left(N+N^{2}\right) \alpha_{n} .
$$

Therefore

$$
\lambda_{1}(\Omega) \leq \frac{\|\nabla f\|_{2}^{2}}{\|f\|_{2}^{2}} \leq N^{\prime} \frac{\alpha_{n}}{\beta_{n}},
$$

where $N^{\prime}=N+N^{2}$, and since

$$
\frac{|\partial \Omega|}{|\Omega|}=\frac{\alpha_{n}}{\beta_{n}} \geq \frac{1}{2}
$$

one gets

$$
\lambda_{1}(\Omega) \leq 2 N^{\prime}\left(\frac{|\partial \Omega|}{|\Omega|}\right)^{2} .
$$

Assume now that $\beta_{n-1}>\alpha_{n}$. Let $f=\sum_{i=1}^{n} f_{i} 1_{\Omega_{i}}$, where $f_{i}=1$ for $1 \leq i \leq p$, $f_{i}-f_{i+1}=\frac{1}{n-p}$ for $p \leq i \leq n$, and $p$ is to be chosen later. One has

$$
\|f\|_{2}^{2}=\sum_{i=1}^{n} f_{i}^{2} \alpha_{i} \geq \beta_{p}
$$

On the other hand

$$
\|\nabla f\|_{2}^{2}=\sum_{i=1}^{n} \sum_{x \in \Omega_{i}}|\nabla f(x)|^{2}+\sum_{x \in X \backslash \Omega}|\nabla f(x)|^{2} .
$$

Let us estimate $|\nabla f(x)|^{2}=\sum_{y \in X, x \sim y}|f(x)-f(y)|$. If $x \in \Omega_{i}, 1 \leq i \leq p-1,|\nabla f(x)|=0$. If $x \in \partial \Omega_{i}, p \leq i \leq n$, use hte fact that $x$ has at most $N$ neighbours $y$. If $y \in \Omega_{i}$, $|f(x)-f(y)|=\overline{0}$; otherwise $y \in \Omega_{i-1} \cup \Omega_{i+1}$, and $|f(x)-f(y)| \leq \frac{1}{n-p}$. Thus

$$
\sum_{x \in \Omega_{i}}|\nabla f(x)|^{2} \leq \frac{N \alpha_{i}}{(n-p)^{2}}
$$

One gets in the same way

$$
\sum_{x \in X \backslash \Omega}|\nabla f(x)|^{2} \leq \frac{N^{2} \alpha_{n}}{(n-p)^{2}}
$$

so that

$$
\|\nabla f\|_{2}^{2} \leq \frac{N^{\prime} \sum_{i=p}^{n} \alpha_{i}}{(n-p)^{2}}
$$


To prove the claim, we have to choose $p \in\{1, \ldots, n-1\}$ such that

$$
\frac{N^{\prime} \sum_{i=p}^{n} \alpha_{i}}{\beta_{p}(n-p)^{2}} \leq C \frac{\alpha_{n}^{2}}{\beta_{n}^{2}},
$$

i.e.

$$
\beta_{n}^{2} \sum_{i=p}^{n} \alpha_{i} \leq \frac{C}{N^{\prime}} \beta_{p}(n-p)^{2} \alpha_{n}^{2} .
$$

Since $\alpha_{i} \leq K \alpha_{n}, \sum_{i=p}^{n} \alpha_{i} \leq K(n-p+1) \alpha_{n} \leq 2 K(n-p) \alpha_{n}$, therefore it suffices to get

$$
\beta_{n}^{2} \leq \frac{C}{2 K N^{\prime}}(n-p) \beta_{p} \alpha_{n}
$$

If $\beta_{1}=\alpha_{1}>(n-1) \alpha_{n}$, then one easily gets (4.1) for $p=1$. Otherwise, one can define

$$
p=\max \left\{i=1, \ldots, n-2 ; \beta_{i} \leq(n-i) \alpha_{n}\right\}+1
$$

Then

$$
\beta_{p-1} \leq(n-p+1) \alpha_{n}
$$

therefore, since $\alpha_{p} \leq K \alpha_{n}$,

$$
\beta_{p}=\beta_{p-1}+\alpha_{p} \leq K(n-p+2) \alpha_{n}
$$

(one can always assume $K \geq 1$ ). On the other hand, since $\beta_{n-1}>\alpha_{n}$, one certainly has

$$
\beta_{p}>(n-p) \alpha_{n}
$$

Now $n-p+2 \leq 3(n-p)$, and

$$
(n-p) \alpha_{n} \leq \beta_{p} \leq 3 K(n-p) \alpha_{n},
$$

whence

$$
\beta_{n}^{2} \leq\left(\beta_{p}+K(n-p) \alpha_{n}\right)^{2} \leq 16 K^{2}(n-p)^{2} \alpha_{n}^{2} \leq 16 K^{2}(n-p) \beta_{p} \alpha_{n}
$$

The proposition is proved with $C=32 K N^{\prime}$.

One easily deduces from 4.9

4.10 Proposition Let $\Omega$ be a finite subset of $X$. Define $\partial_{i} \Omega=\left\{x \in \Omega ; d\left(x, \Omega^{c}\right)=i\right\}$. Let $p$ be the smallest integer such that $\partial_{p+1} \Omega=\emptyset$. Then, if $\forall i=1, \ldots, p,\left|\partial_{i} \Omega\right| \leq K|\partial \Omega|$,

$$
\lambda_{1}(\Omega) \leq C\left(\frac{|\partial \Omega|}{|\Omega|}\right)^{2},
$$

where $C$ only depends on the constants of the graph $X$ and on $K$.

Let us say that $\Omega$ is $K$-admissible if it satisfies the assumptions of Proposition 4.10. We can now state 
4.11 Corollary. Suppose that $\gamma$ satisfies $(D)$, that $\varphi$ is non-decreasing and that they are related by $\gamma^{\prime}(t)=\frac{\gamma(t)}{\varphi^{2}(\gamma(t))}$ or $t=\int_{0}^{\gamma(t)} \frac{\varphi^{2}(v)}{v} d v$. If there exists a family of $K$-admissible sets $\Omega_{\xi}, \xi \in \mathbb{R}_{+}^{*}$ such that such that $\left|\Omega_{\xi}\right| \leq \xi$ and $\frac{\left.a b s \partial \Omega_{\xi}\right)}{\left.a b s \Omega_{\xi}\right)} \leq \frac{1}{\varphi(\xi)}$, then

$$
\sup _{x \in X} p_{k}(x, x) \geq \frac{1}{\gamma(C k)}, \forall k \in \mathbb{N}^{*} \text {. }
$$

\section{Exponential decay}

Let $(X, \mu)$ be a $\sigma$-finite measured space, and $T_{t}$ a semi-group of symmetric contractions of of $L^{2}(X, \mu)$. Denote by $-A$ the infinitesimal generator of $T_{t}$ and $p_{t}$ its kernel when it exists $($ see $\S 2)$. Suppose $\lambda_{0}=\inf _{f \in \mathcal{D}} \frac{(A f, f)}{\|f\|_{2}^{2}}>0$. The above techniques also work in that setting; however, they are less interesting because the corresponding results on upper bounds are far from being complete. One deduces from 2.4

\subsection{Proposition.}

$$
\sup _{x \in X} p_{t}(x, x) \geq e^{-1} e^{-\lambda_{0} t} \sup \left\{\frac{1}{|\Omega|} ; \lambda_{1}(\Omega) \leq \lambda_{0}+\frac{1}{t}\right\} \text {. }
$$

5.2 Theorem. Suppose that for some $t \in \mathbb{R}_{+}^{*}$, there exists $\Omega_{t}$ such that $\lambda_{1}\left(\Omega_{t}\right) \leq \lambda_{0}+\frac{1}{t}$ and $\left|\Omega_{t}\right| \leq v$. Then $\sup _{x \in X} p_{t}(x, x) \geq e^{-\lambda_{0} t} / e v$.

One proves as in 3.2

5.3 Theorem. Let $\gamma$ be an increasing $C^{1}$ bijection of $\mathbb{R}_{+}^{*}$ satisfying $(D)$. Let $\Lambda$ be such that $\gamma^{\prime}(t)=\gamma(t) \Lambda(\gamma(t))$ or $t=\int_{0}^{\gamma(t)} \frac{d v}{v \Lambda(v)}$. If for every $\xi \in \mathbb{R}_{+}^{*}$, there exists $\Omega_{\xi}$ such that $\left|\Omega_{\xi}\right| \leq \xi$ and $\lambda_{1}\left(\Omega_{\xi}\right) \leq \lambda_{0}+\Lambda(\xi)$, then

$$
\sup _{x \in X} p_{t}(x, x) \geq \frac{e^{-\lambda_{0} t}}{\gamma(c t)}, \forall t>0 .
$$

We now give partial results concerning upper bounds. The following is an adaptation of [C1], §IV:

5.4 Theorem. Let $T_{t}$ be as above, and $\left.\varepsilon \in\right] 0,1[$. Then

$$
\sup _{x \in X} p_{t}(x, x) \leq C e^{-\lambda_{0} t} t^{-\varepsilon}, \forall t \geq 1
$$

if and only if

$$
\|f\|_{\infty} \leq C\|f\|_{2}^{1-\varepsilon}\left\|\left(A-\lambda_{0}\right)^{1 / 2} f\right\|_{2}^{\varepsilon}, \forall f \in L^{2} \cap \mathcal{D}\left(A^{1 / 2}\right)
$$

such that $\left\|\left(A-\lambda_{0}\right)^{1 / 2} f\right\|_{2} \leq\|f\|_{2}$.

One deduces immediately from Theorem 5.4 that, if $T_{t}$ is as above, if $\left.\varepsilon \in\right] 0,1[$ and

$$
\sup _{x \in X} p_{t}(x, x) \leq C e^{-\lambda_{0} t} t^{-\varepsilon}, \forall t \geq 1 \text {, }
$$

then

$$
\lambda_{1}(\Omega) \geq \lambda_{0}+c|\Omega|^{-\frac{1}{\varepsilon}},
$$

as soon as $|\Omega|$ is large enough. We are going to show that this implication also holds for $\varepsilon>1$. 
5.5 Theorem. Let $T_{t}$ be as above, and $\varepsilon>0$. Then

$$
\sup _{x \in X} p_{t}(x, x) \leq C e^{-\lambda_{0} t} t^{-\varepsilon}, \forall t \geq 1
$$

implies

$$
\lambda_{1}(\Omega) \geq \lambda_{0}+c|\Omega|^{-\frac{1}{\varepsilon}},
$$

as soon as $\Omega$ is large enough.

Proof: According to 2.3 and the hypothesis,

$$
\frac{1}{|\Omega|} \exp \left(-\lambda_{1}(\Omega) t\right) \leq C e^{-\lambda_{0} t} t^{-\varepsilon}, \forall t \geq 1, \forall \Omega,
$$

therefore

$$
\lambda_{1}(\Omega) \geq \frac{1}{t} \log \left(\frac{e^{\lambda_{0} t} t^{\varepsilon}}{C|\Omega|}\right)=\lambda_{0}+\frac{1}{t} \log \left(\frac{t^{\varepsilon}}{C|\Omega|}\right) .
$$

The result follows by choosing $t=(e C|\Omega|)^{1 / \varepsilon} . \square$

For example, on a co-compact covering manifold whose deck transformation group is non amenable, an argument by T. Lyons shows that the hypothesis of 5.4 holds with $\varepsilon=1$ (see the appendix of $[\mathrm{CCMP}]$ ). Therefore, for $\Omega$ large,

$$
\lambda_{1}(\Omega) \geq \lambda_{0}+c|\Omega|^{-1} .
$$

One can of course wonder whether the converse of 5.4 holds. This is not likely to be true, since for example if $X$ is the hyperbolic space,

$$
\sup _{x \in X} p_{t}(x, x) \leq C e^{-\lambda_{0} t} t^{-3 / 2}, \forall t \geq 1
$$

whereas one can check that the lower bound on $\lambda_{1}(\Omega)$ is much better that the one given by 5.5 (roughly $\lambda_{1}(\Omega) \geq \lambda_{0}+\frac{c}{\log |\Omega|}$ ).

The above results have discrete time analogues, in the spirit of [C1], $\S \mathrm{V}$ and $\S 4$ above. We leave the details to the reader.

Let us however point out the following facts. Let $X=\Gamma$ be a non-amenable finitely generated group and $\mu$ a symmetric probability measure on $\Gamma$ with finite and generating support. Let $T$ the right convolution operator on $\ell^{2}(\Gamma)$ associated with $\mu$, and let $a=$ $\|T\|_{2 \rightarrow 2}$; note that $a<1$ since $\Gamma$ is non-amenable. It was remarked by Guivarc'h $([\mathrm{Gu}]$, see also [La], prop. 2.1) that

$$
\mu^{(2 k)}(\{e\})=\left\|T^{2 k}\right\|_{1 \rightarrow \infty} \leq C a^{2 k} k^{-1}
$$

Using Proposition 4.2, one can as above deduce from this estimate that

$$
a(\Omega) \leq a e^{-c|\Omega|^{-1}}, \forall \Omega \text { finite subset of } \Gamma .
$$


In the case where $\Gamma$ is the free group, since

(see $[\mathrm{La}])$, one gets

$$
\mu^{(2 k)}(\{e\})=\left\|T^{2 k}\right\|_{1 \rightarrow \infty} \leq C a^{2 k} k^{-3 / 2},
$$

$$
a(\Omega) \leq a e^{-c|\Omega|^{-2 / 3}} .
$$

For comparison, in the amenable case, when $\Gamma$ has polynomial growth of order $D$, this formulation of the Faber-Krahn inequality gives

$$
a(\Omega) \leq e^{-c|\Omega|^{-2 / D}},
$$

and when it has intermediate or exponential growth of order $\alpha \in] 0,1]$,

$$
a(\Omega) \leq e^{-\left(C+C^{\prime} \log |\Omega|\right)^{-1-2 / \alpha}} .
$$

\section{Pointwise lower bounds on general manifolds}

We shall prove here pointwise heat kernel lower bounds under the assumption that we are given an upper bound of the volume function. This hypothesis alone is still not enough to produce any pointwise heat kernel lower bound what will be shown by the examples below. So, we have to introduce additional hypotheses, and we will do that in two ways.

The first way is to assume that we know the geometry of the manifold near a point at which we are looking at the heat kernel. It turns out that apart from the volume upper bound, it suffices to know some geometric characteristics (such as certain isoperimetric constants) in an arbitrarily small neighbourhood of the point.

The second way (which will be taken in the next section) is to assume that we are given a proper upper bound of the heat kernel. The fact is that an upper bound of the heat kernel and that of the volume growth function imply a lower on-diagonal bound for the heat kernel. This idea was exploited in [St], [BCF], [CS2], and [L], but here we implement it in a much more general setting.

In order to state the result of the first kind, we fix a point $z \in M$ and assume that for some $r_{0}>0$ and for any $r \geq r_{0}$ the volume $V(z, r)$ of the ball $B(z, r)$ is bounded from above by a function of the radius:

$$
V(z, r) \leq v(r)
$$

We suppose also that the function $v(r)$ is regular in the following sense: it is a continuous monotonically increasing function on $\left[r_{0},+\infty\right)$ and, in addition, the function

$$
\frac{r^{2}}{\log v(r)}
$$

is strictly increasing on $\left[r_{0},+\infty\right)$ and goes to $\infty$ as $r \rightarrow \infty$. We will always assume that $v\left(r_{0}\right)>2$ in order to keep $\log v\left(r_{0}\right)$ away from 0 .

Let us put

$$
t_{0}=\frac{r_{0}^{2}}{\log v\left(r_{0}\right)}
$$

then the function (6.2) has an inverse defined on $\left[t_{0}, \infty\right)$ which will be denoted by $\mathcal{R}(t)$. In other words, $\mathcal{R}(t)$ satisfies the identity

$$
t=\frac{\mathcal{R}^{2}(t)}{\log v(\mathcal{R}(t))} .
$$


6.1 Theorem. Under the above assumptions, the following lower bound for the heat kernel is valid for all $t \geq t_{0}$

$$
p_{t}(z, z) \geq \frac{0.5}{V(z, \mathcal{R}(a t))}
$$

where the constant $a>0$ depends on the interior geometry of the ball $B\left(z, r_{0}\right)$ of center $z$ and radius $r_{0}$ in the following sense:

$$
a=a\left(r_{0}, n, \kappa\left(z, r_{0}\right)\right)
$$

where $n=\operatorname{dim} M$ and $\kappa\left(z, r_{0}\right)$ is the Faber-Krahn isoperimetric constant in the ball $B\left(z, r_{0}\right)$.

\section{Remarks:}

1. The explicit definition of $\kappa\left(z, r_{0}\right)$ is given below in the course of the proof. What is important to underline is the fact that $a$ is determined by an arbitrarily small neighbourhood of the point $z$ and, therefore, does not depend on the global geometry of the manifold in question.

2. The coefficient 0.5 in (6.5) can be replaced by any number $<1$ at the expense of increasing $a$.

3. As follows from (6.5) and (6.1),

$$
p_{t}(z, z) \geq \frac{0.5}{v(\mathcal{R}(a t))}
$$

If we rewrite the identity $(6.4)$ in the form

$$
\log v(\mathcal{R}(a t))=\frac{\mathcal{R}^{2}(a t)}{a t}
$$

we obtain obviously from $(6.7)$

$$
p_{t}(z, z) \geq \frac{1}{2} \exp \left(-\frac{\mathcal{R}^{2}(a t)}{a t}\right)
$$

which can be considered as an alternative way of writing (6.5) .

4. Theorem 6.1 applies on any complete manifold provided the volume of a ball is growing, roughly speaking, slower than $e^{c r^{2}}$. Indeed, given a point $z$ and some $r_{0}>0$, a Faber-Krahn type inequality does hold in $B\left(z, r_{0}\right)$ just by compactness of the ball. Therefore, we have some finite value for $a$ by (6.6). On the other hand, if the volume $V(z, r)$ grows essentially slower than $e^{c r^{2}}$ then it has an upper bound function $v(r)$ satisfying the above regularity conditions. Hence, all hypotheses of Theorem 6.1 hold, and so does the lower bound (6.5) .

5. One can slightly generalize Theorem 6.1 by assuming that the function $v(r)$ is defined on an interval $\left[r_{1}, \infty\right)$ where $r_{1}>r_{0}$, and, respectively, the upper bound (6.1) of the volume function is valid only for $r \geq r_{1}$. Then the conclusion (6.5) is true for $t \geq t_{1}$ (where $t_{1}$ is the bottom of the domain of $\mathcal{R}(t))$ with $a=\left(r_{0}, r_{1}, n, \kappa\left(z, r_{0}\right)\right)$.

6 . On a manifold of bounded geometry, for some $r_{0}>0$ and for any $z \in M$ the isoperimetric constant $\kappa\left(z, r_{0}\right)$ is bounded away from zero. Therefore, the constant $a$ can be taken 
the same for all $z$ so that the lower bound (6.5) becomes uniform in $z$ provided there is a uniform upper bound of the volume.

7. If the volume $V(z, r)$ grows as $r \rightarrow \infty$ essentially faster than $e^{c r^{2}}$ then the manifold may be stochastically incomplete (see $[\mathrm{Az}],[\mathrm{G} 5]$ ). Our method of proof cannot handle such a situation.

8. In the setting of Markov chains on graphs (and even in a more general operator theoretic setting), Françoise Lust-Piquard already obtained in [L] essentially the analogue of Theorem 6.1 (see below for some details). We owe her the observation that our method works in this setting also, provided that instead of the integrated maximum principle (i.e. the fact that $E(z, t)$ is non-increasing in $t)$, one estimates directly $\sum_{y \in X \backslash B(x, R)} p_{k}(x, y)$ (see $\S 4$ for notation) using the transmutation formula of [Carn] as in [L]. One could also use the universal Gaussian estimate for Markov chains ([V1], [Carn]).

\section{Examples:}

1. If we take

$$
v(r)=C r^{N}
$$

then we have

$$
\mathcal{R}(t) \sim \sqrt{t \log t}
$$

and Theorem 6.1 yields

$$
p_{t}(z, z) \geq \frac{0.5}{V(z, \sqrt{a t \log a t})} \geq \frac{\text { const }}{(t \log t)^{\frac{N}{2}}} .
$$

As will be shown in $\S 9$, one cannot get an essentially better lower bound without assuming anything else.

In particular, if $M$ satisfies the doubling volume property around $z$, i.e.

$$
V(z, 2 r) \leq \mathrm{const} V(z, r), \forall r>0
$$

then one can indeed take $v(r)=C r^{N}$. The bound $V(z, r) \leq C r^{N}$ may be very rough, but the estimate

$$
p_{t}(z, z) \geq \frac{0.5}{V(z, \sqrt{a t \log a t})}
$$

still holds.

Let us note that F.Lust-Piquard [L] obtained similar estimates in the discrete time setting.

2. For an exponential volume growth

$$
v(r)=\exp \left(r^{\alpha}\right)
$$

where $\alpha \in(0,2)$, we have

$$
\mathcal{R}(t) \sim t^{\frac{1}{2-\alpha}}
$$

and, thus,

$$
p_{t}(z, z) \geq \text { const } \exp \left(-\operatorname{const} t^{\frac{\alpha}{2-\alpha}}\right) .
$$

An example in $\S 8$ will show that the exponent $t^{\frac{\alpha}{2-\alpha}}$ is sharp whenever $\alpha \leq 1$. This is better that the exponent $t^{\frac{\alpha(1+\alpha)}{2}}$ obtained in a discrete setting in $[\mathrm{L}]$ for $\alpha \in(0,1]$. However, after 
reading a first version of the present paper, Françoise Lust-Piquard noticed that her own argument could be modified in order to get the correct exponent (namely, in the proof of her Theorem 3, in the case iii), one should sum as below over a series of annuli instead of the exterior of a ball).

Proof of Theorem 6.1. We start with the following lemma which is the core of all on-diagonal lower bounds. In the lemma below, we do not assume anything about the manifold except for what is stated explicitly. We denote the Riemannian volume of a set $\Omega \subset M$ by $\mu(\Omega)$ or $|\Omega|$.

6.2 Lemma. Let the manifold $M$ be stochastically complete and let $z$ be a point in $M$. Let us fix a time $t>0$ and suppose that for some region $\Omega \subset M$

$$
\int_{M \backslash \Omega} p_{t / 2}(z, x) d x<\varepsilon
$$

where $\varepsilon \in(0,1)$. Then

$$
p_{t}(z, z) \geq \frac{(1-\varepsilon)^{2}}{|\Omega|}
$$

Proof of the lemma. The idea behind the proof is classical (see for example [St], lecture III, [BCF], [CS2], and [L]).

The proof uses two crucial properties of the heat kernel:

$1^{\circ}$ The semigroup property which yields, in particular,

$$
p_{t}(z, z)=\int_{M} p_{t / 2}^{2}(z, x) d x
$$

$2^{\circ}$ The hypothesis of stochastic completeness which means that for all $z \in M, t>0$ (or, which is the same, for some $z \in M, t>0$ )

$$
\int_{M} p_{t}(z, x) d x=1
$$

We have by (6.10)

$$
\begin{aligned}
p_{t}(z, z) & =\int_{M} p_{t / 2}^{2}(z, x) d x \geq \int_{\Omega} p_{t / 2}^{2}(z, x) d x \\
& \geq \frac{1}{|\Omega|}\left(\int_{\Omega} p_{t / 2}(z, x) d x\right)^{2} \\
& =\frac{1}{|\Omega|}\left(1-\int_{M \backslash \Omega} p_{t / 2}(z, x) d x\right)^{2}
\end{aligned}
$$

whence (6.9) follows.

To prove Theorem 6.1 , we take $\Omega=B(z, R)$ where $R \geq r_{0}$ will be found to satisfy (6.8) for some $\epsilon \in(0,1)$. Eventually, $R$ will be equal to $\mathcal{R}(a t)$ for some (big) constant $a$. 
Let us note that under the hypotheses of Theorem 6.1 the manifold is stochastically complete so we can apply Lemma 6.2. Indeed, the hypothesis that the function $r^{2} / \log v(r)$ is increasing implies $v(r) \leq e^{C r^{2}}$ and $V(z, r) \leq e^{C r^{2}}$ for large $r$, which, in turn, implies stochastic completeness by [G1] or [KL].

In order to estimate the integral (6.8), we shall use the following quantity

$$
E(z, t)=\int_{M} p_{t}^{2}(z, x) \exp \left(\frac{d^{2}}{4 t}\right) d x
$$

(where $d=\operatorname{dist}(z, x)$ ) which is known to be finite on any manifold and, moreover, is a decreasing function of $t$ (see [G2] and [G3]). We have by the Cauchy-Schwarz inequality

$$
\begin{gathered}
\left(\int_{M \backslash B(z, R)} p_{t / 2}(z, x) d x\right)^{2} \leq \int_{M \backslash B(z, R)} p_{t / 2}^{2}(z, x) e^{\frac{d^{2}}{2 t}} d x \int_{M \backslash B(z, R)} e^{-\frac{d^{2}}{2 t}} d x \\
\leq E(z, t / 2) \int_{M \backslash B(z, R)} e^{-\frac{d^{2}}{2 t}} d x
\end{gathered}
$$

where $d=\operatorname{dist}(z, x)$.

Let us introduce the notation ${ }^{\dagger}$

$$
I(z, R)=\int_{M \backslash B(z, R)} e^{-\frac{d^{2}}{2 t}} d x
$$

so that we can write down the following inequality

$$
\left(\int_{M \backslash \Omega} p_{t / 2}(z, x) d x\right)^{2} \leq E(z, t / 2) I(z, R)
$$

which is true without any a priori assumptions. We will estimate from above each term on the right hand side of $(6.12)$.

Let us first estimate $I(z, R)$ from above by decomposing it into a series of integrals over the annuli. Namely, we take a sequence $R_{k}=2^{k} R, k=0,1,2 \ldots$ and write

$$
\begin{aligned}
I(z, R) & \leq \sum_{k=0}^{\infty} \exp \left(-\frac{R_{k}^{2}}{2 t}\right) \mu\left(B\left(z, R_{k+1}\right) \backslash B\left(z, R_{k}\right)\right) \\
& \leq \sum_{k=0}^{\infty} \exp \left(-\frac{R_{k}^{2}}{2 t}\right) V\left(z, R_{k+1}\right) \\
& \leq \sum_{k=0}^{\infty} \exp \left(-\frac{R_{k+1}^{2}}{8 t}+\log v\left(R_{k+1}\right)\right) .
\end{aligned}
$$

The idea of our further estimates is that the first (negative) term in the exponent overrides the second (positive) one which ensures an upper bound for $I(z, R)$. To make it rigorous, let us take

$$
R=\mathcal{R}(a t)
$$

$\dagger$ Let us note that $I(z, R)$ depends on $t$ as well but we suppress that in our notation in order not to overload it. 
for some (big) $a>1$ to be found later. It means that $R \geq r_{0}$ and

$$
\frac{R^{2}}{\log v(R)}=a t
$$

whence it follows

$$
\frac{R_{k+1}^{2}}{\log v\left(R_{k+1}\right)} \geq a t
$$

(because the function $r^{2} / \log v(r)$ is increasing in $r$ ) or

$$
\log v\left(R_{k+1}\right) \leq \frac{R_{k+1}^{2}}{a t}
$$

Let us further suppose that

$$
a \geq 16
$$

which gives us from (6.16)

$$
-\frac{R_{k+1}^{2}}{8 t}+\log v\left(R_{k+1}\right) \leq\left(-1+\frac{8}{a}\right) \frac{R_{k+1}^{2}}{8 t} \leq-\frac{1}{2} \frac{R_{k+1}^{2}}{8 t}
$$

Therefore, we obtain the following upper bound of $I(z, R)$ :

$$
I(z, R) \leq \sum_{k=0}^{\infty} \exp \left(-\frac{R_{k+1}^{2}}{16 t}\right)
$$

Substituting here $R_{k+1}=2^{k+1} R$ and $R^{2} / t=a \log v(R)$ from (6.14), we obtain

$$
I(z, R) \leq \sum_{k=0}^{\infty} \exp \left(-4^{k} \frac{a}{4} \log v(R)\right)
$$

or, by using $4^{k} \geq k+1$ and changing $k+1$ to $k$

$$
I(z, R) \leq \sum_{k=1}^{\infty} v(R)^{-\frac{a}{4} k}=\frac{1}{v(R)^{a / 4}-1} \leq \frac{1}{v\left(r_{0}\right)^{a / 4}-1}
$$

To estimate $E(z, t / 2)$ we use the fact that it is decreasing in $t$ whence we have for $t \geq t_{0}$

$$
E(z, t / 2) \leq E\left(z, t_{0} / 2\right)
$$

Returning to (6.12), we have for $R=\mathcal{R}($ at $)$ and $t \geq t_{0}$

$$
\left(\int_{M \backslash B(z, R)} p_{t / 2}(z, x) d x\right)^{2} \leq \frac{E\left(z, t_{0} / 2\right)}{v\left(r_{0}\right)^{a / 4}-1} .
$$


Let us take $a$ large enough to ensure that the right hand side in (6.18) is less than a given $\varepsilon^{2}$. Namely, we can take any (virtually, the smallest) $a$ such that

$$
a \geq 16+4 \frac{\log \left(1+\varepsilon^{-2} E\left(z, t_{0} / 2\right)\right)}{\log v\left(r_{0}\right)}
$$

and for that $a$ and for any $t \geq t_{0}$ we have by Lemma 6.2

$$
p_{t}(z, z) \geq \frac{(1-\varepsilon)^{2}}{V(z, \mathcal{R}(a t))}
$$

For a suitable $\varepsilon$ one obtains (6.5) .

Let us discuss what our $a$ depends on. If fact, what counts is an upper bound for $a$. Therefore, we have to obtain an upper bound of $E\left(z, t_{0} / 2\right)$. Let us note that in any ball $B(x, \rho)$ the geometry of the manifold is close to that of the Euclidean space. In particular, a Faber-Krahn type inequality is true: for any region $U \subset B(z, \rho)$

$$
\lambda_{1}(U) \geq \kappa(z, \rho)|U|^{-2 / n}
$$

where $\lambda_{1}(U)$ is the first Dirichlet eigenvalue in $U, \kappa(z, \rho)$ is a positive number ("a FaberKrahn constant"), and $n=\operatorname{dim} M$.

The following statement was proved in [G2], Theorem 6.2.

6.3 Proposition. ([G2]) Suppose that on a complete manifold $M$ for some point $z$, for some number $\rho>0$, and for any precompact region $U \subset B(z, \rho)$

$$
\lambda_{1}(U) \geq \kappa|U|^{-\nu}
$$

with some $\kappa>0$. Then for any $t>0$

$$
E(z, t) \leq \frac{\text { const }_{\nu}}{\kappa^{1 / \nu} \min \left(t, \rho^{2}\right)^{1 / \nu}}
$$

Remark: Let us underline that the validity of (6.22) does not depend on geometry off the $\rho$-neighbourhood of $z$. The key point is that in order to estimate $E(z, t)$ for small $t$ (namely, for $t<\rho^{2}$ ) it suffices to know the geometry of a small neighbourhood of the point $z$. For larger $t$, one simply uses the general fact that $E(z, t)$ is a decreasing function of $t$.

Let us take now $\nu=2 / n, \rho=r_{0}$, and $\kappa=\kappa\left(z, r_{0}\right)$ and obtain from (6.22)

$$
E\left(z, t_{0} / 2\right) \leq \operatorname{const}_{n}\left(\frac{\log v\left(r_{0}\right)}{\kappa\left(z, r_{0}\right) r_{0}^{2}}\right)^{\frac{n}{2}}
$$

(we replaced here $t_{0}$ by its value from (6.3) using the assumption that $\log v\left(r_{0}\right) \geq \log 2$ ).

Therefore, the number $a$ satisfying (6.19) can be taken as

$$
a=16+\sup _{\xi>0} \frac{4}{\xi} \log \left(1+\operatorname{const}_{n}\left(\frac{\xi}{\kappa r_{0}^{2}}\right)^{\frac{n}{2}}\right)
$$


where we have replaced $\varepsilon$ by its value from $(1-\varepsilon)^{2}=\frac{1}{2}$, put $\xi=\log v\left(r_{0}\right)$, and maximized in $\xi$.

If we set

$$
\eta=\operatorname{const}_{n}\left(\frac{\xi}{\kappa r_{0}^{2}}\right)^{\frac{n}{2}}
$$

we can rewrite $(6.23)$ as follows

$$
a=16+\frac{\text { const }_{n}}{\kappa r_{0}^{2}} \sup _{\eta>0} \frac{\log (1+\eta)}{\eta^{\frac{2}{n}}} .
$$

Since the supremum on the right hand side is finite, we obtain finally

$$
a=16+\frac{\text { const }_{n}}{\kappa r_{0}^{2}}=a\left(r_{0}, n, \kappa\left(z, r_{0}\right)\right)
$$

which finishes the proof.

\section{Manifolds with the doubling volume property}

Now we turn to another kind of result where we will assume an upper bound of the heat kernel. The main motivation is that if one has the doubling volume property, Theorem 6.1 yields

$$
\mathcal{R}(t) \asymp \sqrt{t \log t}
$$

and

$$
p_{t}(z, z) \geq \frac{\text { const }}{V(z, \sqrt{t \log t})}
$$

whereas one might expect the sharper

$$
p_{t}(z, z) \geq \frac{\text { const }}{V(z, \sqrt{t})} .
$$

Indeed, let us recall that in the situation of non-negative Ricci curvature (where the doubling volume property indeed holds) one has by the theorem of Li and Yau [LY] that for all $x \in M$ and $t>0$

$$
\frac{C_{1}}{V(x, \sqrt{t})} \leq p_{t}(x, x) \leq \frac{C_{2}}{V(x, \sqrt{t})}
$$

where $C_{1,2}=C_{1,2}(n)>0$.

As will be explained in the examples below, the lower bound (7.1) cannot be replaced by (7.2) so long as one stays in the setting of Theorem 6.1. In order to get (7.2) and, hence, to be able to recover the non-negative curvature case, we have to make some further hypotheses about the manifold. 
7.1 Theorem. Let us suppose that on a complete manifold $M$ for a fixed point $z$, for all $r>0$,

$$
V(z, r) \leq v(r)
$$

where $v(\cdot)$ is an increasing continuous function such that

$1^{\circ}$ for some positive $r_{0}$ and $A$, we have $v\left(r_{0}\right)>2$ and

$$
v(2 r) \leq A v(r)
$$

for all $r \leq r_{0}$

$2^{\circ}$ for $r \geq r_{0}$, the function

$$
\frac{r^{2}}{\log v(r)}
$$

is increasing in $r$.

Let us suppose also that for some $C>0, T>0$ and for any $t \in(0, T)$

$$
p_{t}(z, z) \leq \frac{C}{v(\sqrt{t})}
$$

Then for some $\varepsilon=\varepsilon(A, C)>0$ and for all positive

$$
t<\min \left(T, \frac{\varepsilon r_{0}^{2}}{\log v\left(r_{0}\right)}\right)
$$

we have

$$
p_{t}(z, z) \geq \frac{c}{v(\sqrt{t})}
$$

where $c=c(A, C)>0$.

If the doubling volume property $1^{\circ}$ holds for all $r>0$ (in other words, we can take $\left.r_{0}=\infty\right)$ the statement can be simplified, and we get the following

7.2 Theorem. Let us suppose that on a complete manifold $M$ for a fixed point $z$, for all $r>0$,

$$
V(z, r) \leq v(r)
$$

where $v(\cdot)$ is an increasing continuous function such that for all $r>0$

$$
v(2 r) \leq A v(r)
$$

for some positive $A$. Let us suppose also that for some $C>0, T>0$ and for any $t \in(0, T)$

$$
p_{t}(z, z) \leq \frac{C}{v(\sqrt{t})}
$$


Then for all $t \in(0, T)$

$$
p_{t}(z, z) \geq \frac{c}{v(\sqrt{t})}
$$

where $c=c(A, C)>0$.

\section{Remarks:}

1. The constant $T$ in Theorem 7.2 may be equal to $\infty$, so if the upper bound (7.9) holds for all $t>0$, then so does the lower bound (7.10). For example, this is the case when the manifold has a non-negative Ricci curvature (see $[\mathrm{LY}]$ ).

2. Suppose $M$ has Ricci curvature bounded below by a (negative) constant $-K$. Fix a point $z \in M$ and some $r_{0}>0$. It is well known that the doubling volume property holds for the balls $B(z, r)$ for $r<r_{0}$, with a constant $A=A\left(r_{0}, K, \operatorname{dim} M\right)$. On the other hand, for large $r$, the volume $V(z, r)$ grows at most exponentially. Therefore, the function $v(r)$ in (7.3) can be taken equal to $V(z, r)$ for $r<r_{0}$ and $e^{\text {const } r}$ for $r>r_{0}$. The upper bound (7.4) was proved by Li-Yau [LY] with $C=C(T, K, \operatorname{dim} M)$. Thus, we get by Theorem 7.1 the lower bound (7.6) . It was also proved in [V3], but our proof has the advantage that it does not make a direct use of the curvature, and, therefore, it is applicable more generally, for instance on a manifold that is quasi-isometric to one with a bounded below Ricci curvature (but in this particular case one can also use the local parabolic Harnack principle that holds on such a manifold - see $[\mathrm{S}]$ ). Mutatis mutandis, the same remarks are in order for Theorem 7.2.

Proof of Theorem 7.1. The approach to the proof is the same as in Theorem 6.1: given $t$ as in (7.5), we will seek a number $R>0$ such that

$$
E(z, t / 2) I(z, R) \leq \frac{1}{2}
$$

which will yield by (6.12) and by Lemma 6.2 that

$$
p_{t}(z, z) \geq \frac{\text { const }}{V(z, R)} \geq \frac{\text { const }}{v(R)} .
$$

Let us note that the manifold in question is stochastically complete because the hypotheses of Theorem 7.1 imply $V(z, r) \leq$ const $e^{\text {const } r^{2}}$. Therefore, Lemma 6.2 is applicable.

In order to estimate $E(z, t / 2)$, we apply the result of [G7] (see Theorem 1.1 there) which states that the upper bound (7.4) alone (with a function $v(r)$ satisfying $1^{\circ}$ ) implies for all positive

$$
t<\min \left(T, r_{0}^{2}\right)
$$

that

$$
E(z, t) \leq \frac{\operatorname{const}_{A, C}}{v(\sqrt{t})}
$$

If $\varepsilon$ is small enough, namely,

$$
\varepsilon<\log 2
$$


then (7.13) follows from (7.5), and so does (7.14).

In order to estimate $I(z, R)$, we use the same decomposition as in the proof of Theorem 6.1 , namely

$$
I(z, R) \leq \sum_{k=0}^{\infty} \exp \left(-\frac{R_{k}^{2}}{2 t}\right) v\left(R_{k+1}\right)
$$

where $R_{k}=2^{k} R$ and

$$
R=a \sqrt{t}
$$

where $a>1$ will be specified later. In fact, we will find first $a=a\left(A, C, v\left(r_{0}\right)\right)$, and only after that $\varepsilon=\varepsilon(A, C)$.

So far we only require that $a$ and $\varepsilon$ are related as follows:

$$
\varepsilon<\frac{\log v\left(r_{0}\right)}{a^{2}}
$$

which ensures by (7.5) that $R<r_{0}$.

Let $N$ be the smallest integer for which

$$
R_{N}>r_{0}
$$

We split the sum in (7.16) into 2 parts: with $k<N$ and with $k \geq N$. In the first part, we have $R_{k} \leq r_{0}$ and, thus, $v\left(R_{k+1}\right) \leq A v\left(R_{k}\right) \leq A^{2} v\left(R_{k-1}\right) \leq \ldots \leq A^{k+1} v(R)$. Therefore,

$$
\begin{aligned}
\sum_{k=0}^{N-1} \exp \left(-\frac{R_{k}^{2}}{2 t}\right) v\left(R_{k+1}\right) & \leq \sum_{k=0}^{N-1} \exp \left(-\frac{R_{k}^{2}}{2 t}\right) A^{k+1} v(R) \\
& \leq v(R) \sum_{k=0}^{\infty} \exp \left(-4^{k} \frac{R^{2}}{2 t}+(k+1) \log A\right) \\
& \leq v(R) \sum_{k=1}^{\infty} \exp \left(-k \frac{a^{2}}{2}+k \log A\right) \\
& \leq v(R) \sum_{k=1}^{\infty} \exp \left(-k \frac{a^{2}}{4}\right) \\
& =\frac{v(R)}{e^{a^{2} / 4}-1}
\end{aligned}
$$

where we have consecutively applied $4^{k} \geq k+1$, changed $k+1$ to $k$, replaced $R$ by $a \sqrt{t}$, and taken $a$ to be so large that

$$
\frac{a^{2}}{4} \geq \log A
$$

To estimate the second part of the sum in question, we first apply the hypothesis $2^{\circ}$ of the theorem in the form

$$
\frac{\log v\left(R_{k+1}\right)}{R_{k+1}^{2}} \leq \frac{\log v\left(r_{0}\right)}{r_{0}^{2}}
$$


which implies

$$
\log v\left(R_{k+1}\right) \leq \frac{R_{k+1}^{2}}{r_{0}^{2}} \log v\left(r_{0}\right) \leq 4^{k+1} \log v\left(r_{0}\right)
$$

Therefore, we have

$$
\begin{aligned}
\sum_{k=N}^{\infty} \exp \left(-\frac{R_{k}^{2}}{2 t}\right) v\left(R_{k+1}\right) & \leq \sum_{k=N}^{\infty} \exp \left(-\frac{R_{k}^{2}}{2 t}+4^{k+1} \log v\left(r_{0}\right)\right) \\
& =\sum_{k=N}^{\infty} \exp \left(-4^{k+1}\left(\frac{a^{2}}{8}-\log v\left(r_{0}\right)\right)\right) \\
& \leq \sum_{k=N}^{\infty} \exp \left(-4^{k+1} \log v\left(r_{0}\right)\right) \\
& \leq \sum_{k=1}^{\infty} \exp \left(-k 4^{N+1} \log v\left(r_{0}\right)\right) \\
& \leq \sum_{k=1}^{\infty} 2^{-k 4^{N+1}} \\
& <2^{-4^{N}} \\
& \leq 2^{-\frac{r_{0}^{2}}{a^{2} t}} .
\end{aligned}
$$

Here we have assumed that the constant $a$ satisfies the inequality

$$
a^{2}>16 \log v\left(r_{0}\right)
$$

then we used the inequality

$$
4^{k+1}=4^{N+1} 4^{k-N} \geq 4^{N+1}(k-N+1)
$$

and changed $k$ to $k-N+1$. Finally, we used that $v\left(r_{0}\right)>2$ and $R_{N}=2^{N} R>r_{0}$ which yields $4^{N}>\frac{r_{0}^{2}}{R^{2}}=\frac{r_{0}^{2}}{a^{2} t}$.

Hence, we have the following upper bound of $I(z, R)$

$$
I(z, R) \leq \frac{v(R)}{e^{a^{2} / 4}-1}+2^{-\frac{r_{0}^{2}}{a^{2} t}}
$$

under the assumptions about $\varepsilon$ and $a$ made above.

Combining this with the upper bound (7.14) of $E(z, t / 2)$ we have by (6.12)

$$
\begin{aligned}
\left(\int_{M \backslash B(z, R)} p_{t / 2}(z, x) d x\right)^{2} & \leq E(z, t / 2) I(z, R) \\
& \leq \frac{\operatorname{const}_{A, C}}{v(\sqrt{t / 2})} \frac{v(R)}{e^{a^{2} / 4}-1}+\frac{\operatorname{const}_{A, C}}{v(\sqrt{t / 2})} 2^{-\frac{r_{0}^{2}}{a^{2} t}}
\end{aligned}
$$

Next we have by the property $1^{\circ}$

$$
\frac{v(R)}{v(\sqrt{t / 2})}=\frac{v(R)}{v(R /(\sqrt{2} a))} \leq A^{\log _{2} a+2}=A^{2} a^{\alpha}
$$


where $\alpha=\log _{2} A$, whence it follows that the first summand on the right hand side of (7.23) does not exceed

$$
\text { const }_{A, C} \frac{a^{\alpha}}{e^{a^{2} / 4}-1}
$$

which is arbitrarily small provided $a \geq \operatorname{const}_{A, C}$ with a large enough constant const $_{A, C}$.

Collecting together all restrictions on $a$ including $(7.20),(7.21)$, and the one above, we can now choose $a$ as follows

$$
a^{2}=\operatorname{const}_{A, C} \log v\left(r_{0}\right)
$$

for a large enough const $_{A, C}$.

Let us estimate the second summand (7.23) by applying the inequality

$$
\frac{1}{v(\sqrt{t / 2})}=\frac{1}{v\left(r_{0}\right)} \frac{v\left(r_{0}\right)}{v(\sqrt{t / 2})} \leq \frac{\operatorname{const}_{A}}{v\left(r_{0}\right)}\left(\frac{r_{0}}{\sqrt{t}}\right)^{\alpha}
$$

which implies that the second term on the right hand side of (7.23) is no bigger than

$$
\frac{\text { const }_{A, C}}{v\left(r_{0}\right)}\left(\frac{r_{0}}{\sqrt{t}}\right)^{\alpha} 2^{-\frac{r_{0}^{2}}{a^{2} t}}
$$

Let us denote $\xi=\frac{r_{0}}{\sqrt{t \log v\left(r_{0}\right)}}$. According to the hypothesis $(7.5)$, we have

$$
\xi \geq \frac{1}{\sqrt{\varepsilon}}
$$

On the other hand, we rewrite $(7.25)$ as

$$
\text { const }_{A, C} \frac{\log ^{\alpha / 2} v\left(r_{0}\right)}{v\left(r_{0}\right)} \xi^{\alpha} 2^{-c \xi^{2}}
$$

where $c=c(A, C)>0$. Note that the two $\log v\left(r_{0}\right)$ - one from the definition of $\xi$ and the other from the definition (7.24) of $a^{2}$ - cancel at the exponent of 2 .

Now it is obvious that by taking small enough $\varepsilon=\varepsilon(A, C)$ we make $\xi$ large by (7.26), and the expression (7.27) becomes arbitrarily small independently of $v\left(r_{0}\right)$. At the same time, we can satisfy our previous assumptions on $\varepsilon$, namely, (7.15) and (7.18) (note, that $\log v\left(r_{0}\right)$ cancels also in $\left.(7.18)\right)$.

Therefore, we have (7.11), and by (7.12)

$$
p_{t}(z, z) \geq \frac{\text { const }}{v(a \sqrt{t})},
$$

whence (7.6) follows upon one more application of the doubling property.

Theorem 7.2 can be obtained either from Theorem 7.1 by an appropriate passage to the limit as $r_{0} \rightarrow \infty$ or directly by a simplified version of the above argument. Let us note that we do not have to treat any longer the case $k>N$. In particular, the constant $a$ depends now only on $A$ and $C$. 
As a bonus, we get under the hypotheses of Theorem 7.2 that

$$
V(z, r) \geq \operatorname{const}_{A, C} v(r)
$$

for any $r \in(0, a \sqrt{T})$. Indeed, by $(7.4),(7.12),(7.17)$ we have for any $t \in(0, T)$

$$
\frac{C}{v(\sqrt{t})} \geq p_{t}(z, z) \geq \frac{\text { const }}{V(z, a \sqrt{t})}
$$

whence (7.29) follows for $r=a \sqrt{t}$.

Of course, it is desirable to have geometric conditions under which the hypotheses of Theorem 7.1 or 7.2 do hold. The next statement provides such conditions in terms of a Faber-Krahn type inequality.

7.3 Corollary. Suppose that for some point $z$, for all $r>0$, and for all precompact regions $U \subset B(z, r)$

$$
\lambda_{1}(U) \geq \frac{\alpha}{r^{2}}\left(\frac{V(z, r)}{|U|}\right)^{\beta}
$$

with positive $\alpha, \beta$. Then we have for all $t>0$ and some $C, c>0$ depending on $\alpha, \beta$

$$
\frac{c}{V(z, \sqrt{t})} \leq p_{t}(z, z) \leq \frac{C}{V(z, \sqrt{t})}
$$

Proof: We claim that (7.30) implies

(a) the doubling volume property: for all $r>0$ and for some $A=A(\alpha, \beta)$

$$
V(z, 2 r) \leq A V(z, r)
$$

(b) the heat kernel upper bound: for all $t>0$ and for some $C=C(\alpha, \beta)$

$$
p_{t}(z, z) \leq \frac{C}{V(z, \sqrt{t})} .
$$

Therefore, we can apply Theorem 7.2 with $v(r)=V(z, r)$ and obtain also for all $t>0$

$$
p_{t}(z, z) \geq \frac{c}{V(z, \sqrt{t})}
$$

with $c=c(\alpha, \beta)>0$. Of course, (a) and (b) imply always the lower bound (7.34) no matter why they do hold. In other words, the upper bound (7.33) is always sharp whenever there is the doubling volume property.

Let us explain how to get (7.32) and (7.33) from (7.30). The upper bound in (7.33) follows immediately from Proposition 6.3. Indeed, given $R>0$, we can rewrite (7.30) in the form $(6.21)$

$$
\lambda_{1}(U) \geq \kappa|U|^{-\nu}
$$


where $\nu=\beta$ and

$$
\kappa=\frac{\alpha}{R^{2}} V(z, R)^{\beta}
$$

Then we have by $(6.22)$

$$
E(z, t) \leq \frac{R^{2 / \beta} \text { const }_{\alpha, \beta}}{V(z, R) \min \left(t, R^{2}\right)^{1 / \beta}}
$$

or

$$
E(z, t) \leq \frac{\text { const }_{\alpha, \beta}}{V(z, R) \min \left(t / R^{2}, 1\right)^{1 / \beta}}
$$

We are left to put here $R=\sqrt{2 t}$ (note that (7.36) is true for all positive $t$ and $R$ ) and to observe the obvious inequality

$$
p_{t}(z, z)=\int_{M} p_{t / 2}^{2}(z, x) d x \leq E(z, t / 2)
$$

which gives us immediately the upper bound in (7.33) .

The doubling volume property (7.32) follows from the following result of Carron (see Proposition 2.4 in [Carr]).

7.4 Proposition. ([Carr]) Suppose that on a complete manifold $M$ for some region $\Omega \subset M$ and for any precompact subregion $U \subset \Omega$

$$
\lambda_{1}(U) \geq \kappa|U|^{-\nu}
$$

with some positive $\kappa, \nu$. Then for any ball $B(x, r) \subset \Omega$

$$
V(x, r) \geq \operatorname{const}_{\nu} \kappa^{1 / \nu} r^{2 / \nu}
$$

where const $_{\nu}>0$.

Indeed, let us apply this proposition in $\Omega=B(z, \rho)$ with some $\rho>0$ and notice that we have the Faber-Krahn constant $\kappa$ in $\Omega$ determined by (7.35) (replace there $R$ by $\rho$ ). Then as follows from (7.37), for any $r<\rho$

$$
V(z, r) \geq \operatorname{const}_{\alpha, \beta}\left(\frac{V(z, \rho)^{\beta}}{\rho^{2}}\right)^{1 / \beta} r^{2 / \beta}
$$

or

$$
V(z, r) \geq \operatorname{const}_{\alpha, \beta}\left(\frac{r}{\rho}\right)^{2 / \beta} V(z, \rho)
$$

whence we get the doubling volume property (7.32) by taking $\rho=2 r$.

Remark: The isoperimetric inequality (7.30) is known to hold on manifolds of nonnegative Ricci curvature (see [G6]). In this case, $z$ may be any point, $\beta=2 / n$ and $\alpha=\alpha(n)$. On the other hand, as proved in [G2] (see Proposition 5.2 there) the assumption that (7.30) holds uniformly for all $z \in M$ is equivalent to the conjunction of the doubling volume property (7.32) and the upper bound (7.33) (again for all $z \in M$ ).

Hence, the hypothesis (7.30) can be regarded as an optimal geometric assumption under which one has the double sided heat kernel estimate (7.31) . 


\section{Upper bounds on rotationally invariant manifolds}

In the next section, we will present some examples which are to show to what extend Theorems 6.1 and 7.1 are sharp. All examples will be manifolds possessing a rotational symmetry. In order to show that a lower bound of the heat kernel is sharp, one has to obtain an upper bound. This is why in this section, we are mainly concerned with heat kernel upper bounds on rotationally invariant manifolds.

We start with a general on-diagonal upper bound, then apply it to a specific manifold. In Proposition 8.1 below, we do not assume a priori anything about the manifold in question except for being a smooth connected Riemannian manifold.

8.1 Proposition. Let $u(x, t)$ be a $C^{2}$ non-negative subsolution to the heat equation for all $x \in M$ and $t>0$, i.e.

$$
u_{t}-\Delta u \leq 0, \quad u(x, t) \geq 0 .
$$

Let we have in addition that

$1^{\circ}$ for all $t>0$

$$
\int_{M} u(x, t) d x \leq 1
$$

$2^{\circ}$ for all $t>0, s>0$ the superlevel set

$$
U_{t, s}=\{x: u(x, t)>s\}
$$

is precompact.

Moreover, let us suppose that for any set $U=U_{t, s}$ there is a Faber-Krahn type isoperimetric inequality

$$
\lambda_{1}(U) \geq \Lambda(|U|)
$$

where $\Lambda(\cdot)$ is a continuous positive decreasing function on $(0,+\infty)$. Then for all $t>0$ and $\delta \in(0,1)$

$$
\int_{M} u^{2}(x, t) d x \leq \frac{2}{\delta \gamma(2(1-\delta) t)}
$$

where the function $\gamma(\cdot)$ is defined from the identity

$$
t=\int_{0}^{\gamma(t)} \frac{d \xi}{\xi \Lambda(\xi)}
$$

(assuming that the integral in (8.4) converges at 0).

Remark: If we take $u(x, t)=p_{t}(z, x)$ and replace $2 t$ by $t$, then (8.3) will give an upper bound

$$
p_{t}(z, z) \leq \frac{2}{\delta \gamma((1-\delta) t)}
$$


This upper bound was proved in [G2] (Theorem 1.1 there) but under the hypothesis that the isoperimetric inequality (8.2) holds for all bounded regions $U$ rather than only for superlevel sets of $u$. In fact, the analysis of the proof [G2] shows that the inequality (8.2) is required only for the sets $U_{t, s}$.

Proof of Proposition 8.1. First of all, we need to replace the function $u(x, t)$ by one with a compact support to avoid difficulties with integration at infinity. To this end, let us introduce a function $\varphi(s)$ being a non-negative, smooth, increasing, and convex function on $[0, \infty)$ so that for some $\varepsilon>0$

$$
\varphi(s) \leq(s-\varepsilon)_{+} .
$$

In fact, we would take the function

$$
\varphi(s)=(s-\varepsilon)_{+}
$$

if it were smooth. The function $\varphi$ which will be used below can be always thought of as a smooth approximation to (8.7) .

Let us put

$$
v(x, t)=\varphi(u(x, t))
$$

so that

$$
v_{t}-\Delta v=\varphi^{\prime}(u)\left(u_{t}-\Delta u\right)-\varphi^{\prime \prime}|\nabla u|^{2} \leq 0
$$

since we have assumed $\varphi^{\prime} \geq 0, \varphi^{\prime \prime} \geq 0$. We want to replace in all our considerations the function $u$ by $v$ and in the end to pass to the limit when $\varepsilon \rightarrow 0$ and $\varphi(s) \uparrow s$. Indeed, all hypotheses of Proposition 8.1 hold for $v$ as well because, as we have shown, $v$ is a non-negative subsolution to the heat equation, then by (8.6) we have $v \leq u$, and, finally, the superlevel sets of $v$ are those of $u$.

Let us take

$$
I(t)=\int_{M} v^{2}(x, t) d x .
$$

This integral is finite because $\operatorname{supp} v(\cdot, t)$ lies in $U_{t, \varepsilon}$ which is compact. We have by the standard Nash argument

$$
\begin{aligned}
I^{\prime}(t)= & 2 \int_{M} v v^{\prime} d x \leq 2 \int_{M} v \Delta v d x \\
& =-2 \int_{M}|\nabla v|^{2} d x .
\end{aligned}
$$

On the other hand, we can estimate the Dirichlet integral $\int_{M}|\nabla v|^{2} d x$ from below by means of (8.2) . Indeed, for any $s>0$ we have

$$
v^{2} \leq(v-s)_{+}^{2}+2 s v
$$

whence

$$
I(t) \leq \int_{\{v>s\}}(v-s)^{2} d x+2 s \int_{M} v d x
$$




$$
\leq \frac{\int_{\{v>s\}}|\nabla(v-s)|^{2} d x}{\lambda_{1}(\{v(x, t)>s\})}+2 s
$$

where we have applied (8.1) and the variational property of the first Dirichlet eigenvalue. Next we note that

$$
\mu(\{v(x, t)>s\}) \leq s^{-1} \int_{M} v d x \leq s^{-1}
$$

which implies by (8.2)

$$
\lambda_{1}(\{v(x, t)>s\}) \geq \Lambda\left(s^{-1}\right)
$$

and

$$
\begin{gathered}
I(t) \leq \frac{\int_{M}|\nabla v|^{2} d x}{\Lambda\left(s^{-1}\right)}+2 s \\
\int_{M}|\nabla v|^{2} d x \geq(I(t)-2 s) \Lambda\left(s^{-1}\right) .
\end{gathered}
$$

Therefore, we obtain

$$
I^{\prime}(t) \leq-2(I(t)-2 s) \Lambda\left(s^{-1}\right)
$$

which is true for any $s>0$. Let us take

$$
2 s=\delta I(t)
$$

for some $\delta \in(0,1)$. We get from $(8.8)$

$$
I^{\prime}(t) \leq-2(1-\delta) I \Lambda\left(\frac{2}{\delta I}\right)
$$

Now we can solve the differential inequality (8.9) by dividing it by $I \Lambda\left(\frac{2}{\delta I}\right)$ and by integrating it over the interval $\left[t_{0}, t\right]$ where $0<t_{0}<t$

$$
\int_{t_{0}}^{t} \frac{I^{\prime} d t}{I \Lambda\left(\frac{2}{\delta I}\right)} \leq-2(1-\delta)\left(t-t_{0}\right)
$$

which yields after the change $\xi=\frac{2}{\delta I}$

$$
\int_{\frac{2}{\delta I\left(t_{0}\right)}}^{\frac{2}{\delta I(t)}} \frac{d \xi}{\xi \Lambda(\xi)} \geq 2(1-\delta)\left(t-t_{0}\right)
$$

Letting $t_{0} \downarrow 0$, we obtain

$$
\int_{0}^{\frac{2}{\delta I(t)}} \frac{d \xi}{\xi \Lambda(\xi)} \geq 2(1-\delta) t .
$$

Comparison with the definition $(8.4)$ of the function $\gamma(\cdot)$ implies that

$$
\frac{2}{\delta I(t)} \geq \gamma(2(1-\delta) t)
$$




$$
\int_{M} v^{2}(x, t) d x \leq \frac{2}{\delta \gamma(2(1-\delta) t)}
$$

We are left to pass to the function $u$ in this inequality. It is obvious that there exist a sequence of functions $\varphi_{k}, k=1,2, \ldots$ satisfying all the conditions above, and $\varphi_{k}(s) \uparrow s$ (with the corresponding $\varepsilon_{k} \downarrow 0$ ) so that we have a sequence of the functions $v_{k}=\varphi_{k}(u) \uparrow u$ as $k \rightarrow \infty$. Since the estimate (8.10) holds for any $v_{k}$ then, by passing to the limit, the same is valid for $u$.

Next we consider a rotationally invariant manifold $M$ which is topologically $\mathbb{R}^{n}$. Let us fixed a point $z \in \mathbb{R}^{n}$, and define the Riemannian metric in the polar coordinates $(r, \theta)$ centered at the point $z$, in the following way

$$
d s^{2}=d r^{2}+h^{2}(r) d \theta^{2} .
$$

The function $h(r)$ is a smooth positive function which is equal to $r$ in a small neighbourhood of 0 so that near the point $z$ the metric is exactly Euclidean.

The formula (8.11) means that the distance in $M$ in the radial direction (i.e. the distance from the point $z$ ) is exactly a Euclidean one, whereas the metric to the angular direction at a point $x$ is proportional to the Euclidean one with the coefficient $h(r) / r$ where $r=$ $\operatorname{dist}(x, z)$. Therefore, we can find the area of any sphere $\partial B(z, r)$ as

$$
S(r)=\omega_{n} r^{n-1}\left(\frac{h(r)}{r}\right)^{n-1}
$$

or

$$
S(r)=\omega_{n} h^{n-1}(r)
$$

(where $\omega_{n}$ is the area of the unit sphere in $\mathbb{R}^{n}$ ).

This shows that off a small neighbourhood of 0 , the function $S(r)$ can be chosen arbitrarily so long as it is positive and smooth. In fact, in our examples, we will work with the volume function

$$
V(r)=|B(z, r)|=\int_{0}^{r} S(\xi) d \xi
$$

which, therefore, can be taken also arbitrarily (away from 0) with the only restriction to be positive, smooth, and with a positive derivative.

The only additional restriction we impose now on the function $V(\cdot)$ is

$$
V(\infty)=\infty
$$

i.e. the total volume of the manifold is infinite.

Our main goal is the heat kernel at the pole $z$, namely, $p_{t}(z, z)$. Let us first estimate it from above by using Proposition 8.1. To that end, we put $u(x, t)=p_{t}(z, x)$ as was suggested in the remark after Proposition 8.1. In order to apply that proposition, we need to know the level sets of $u(x, t)$.

Let us first observe that this function is rotationally symmetric (which follows from the fact that we deal with the minimal heat kernel $\left.p_{t}(z, x)\right)$ i.e. $u(x, t)$ depends only on 
$r=\operatorname{dist}(z, x)$ and $t$. To simplify notations, we will often write $u(r, t)$ instead of $u(x, t)$ assuming $r=\operatorname{dist}(x, z)$.

As was proved in [ChY] (see Lemma 2.3 there), the function $u(r, t)$ is decreasing in $r$. Therefore, any superlevel set $U_{s, t}$ of this function is necessarily a ball centered at the point $z$, or the whole manifold if $\inf _{x \in M} u(x, t)>0$. But the latter cannot happen since the volume of the manifold was assumed to be infinite whereas the function $u(\cdot, t)$ has a finite integral. Thus, the sets $U_{s, t}$ are exactly the balls, and in order to be able to apply Proposition 8.1, we have to estimate from below the first eigenvalue of any ball $B(z, R)$.

8.2 Lemma. For any ball $B(z, R)$ on any rotationally invariant manifold $M$ the following is true

$$
\lambda_{1}(B(z, R)) \geq \frac{1}{4}\left(\inf _{r \leq R} \frac{S(r)}{V(r)}\right)^{2}
$$

Remark: Since

$$
\frac{S(r)}{V(r)} \sim \frac{n}{r} \rightarrow \infty
$$

as $r \rightarrow 0$, then the right hand side of (8.12) is strictly positive.

Proof: In fact, the inequality (8.12) is similar to the Cheeger inequality [Ch], and we use the same argument for the proof.

Let $\varphi(x)$ denote the first Dirichlet eigenfunction in the ball $B(z, R)$ so that we have

$$
\lambda_{1}(B(z, R))=\frac{\int_{B(z, R)}|\nabla \varphi|^{2} d x}{\int_{B(z, R)} \varphi^{2} d x} .
$$

Since the first eigenfunction is unique (up to a multiplicative constant), then it must be rotationally invariant, too i.e. we can write $\varphi(x)=\varphi(r)$. By the maximum principle, $\varphi(r)$ is decreasing in $r$. Therefore, the superlevel sets of $\varphi(r)$ are the balls with the center $z$ as well.

Let us put

$$
\psi=\varphi^{2}
$$

and note

$$
\frac{\int|\nabla \psi| d x}{\int|\psi| d x}=\frac{\int 2 \varphi|\nabla \varphi|}{\int \varphi^{2}} \leq \frac{2\left(\int \varphi^{2} \int|\nabla \varphi|^{2}\right)^{\frac{1}{2}}}{\int \varphi^{2}}=2\left(\frac{\int|\nabla \varphi|^{2}}{\int \varphi^{2}}\right)^{\frac{1}{2}}
$$

whence it follows

$$
\lambda_{1}(B(z, R)) \geq \frac{1}{4}\left(\frac{\int|\nabla \psi|}{\int \psi}\right)^{2} .
$$

To estimate the right hand side integrals in (8.13), let us consider the superlevel sets of the function $\psi$

$$
\Omega_{s}=\{x \in M: \psi(x)>s\}
$$


and apply the coarea formula

$$
\int|\nabla \psi| d x=\int_{0}^{\infty}\left(\int_{\partial \Omega_{s}} 1 d \sigma\right) d s=\int_{0}^{\infty} \operatorname{Area}\left(\partial \Omega_{s}\right) d s .
$$

As follows from above any set $\Omega_{s}$ is a ball centered at the point $z$ of some radius $\rho \leq R$. Obviously, we have

$$
\operatorname{Area}\left(\partial \Omega_{s}\right)=S(\rho) \geq \inf _{r \leq R} \frac{S(r)}{V(r)} V(\rho)=\inf _{r \leq R} \frac{S(r)}{V(r)}\left|\Omega_{s}\right|
$$

which implies

$$
\int|\nabla \psi| d x \geq \inf _{r \leq R} \frac{S(r)}{V(r)} \int_{0}^{\infty}\left|\Omega_{s}\right| d s=\inf _{r \leq R} \frac{S(r)}{V(r)} \int \psi d x
$$

and together with (8.13) gives (8.12) .

As a consequence of Proposition 8.1 and Lemma 8.2, we obtain the following upper bound for the heat kernel.

8.3 Theorem. Let the manifold $M$ be rotationally invariant and of infinite volume. Let also the function

$$
\frac{S(r)}{V(r)}
$$

be nonincreasing on $(0, R)$. Then for any $t \in(0, T)$ and any $\delta \in(0,1)$

$$
p_{t}(z, z) \leq \frac{2(1-\delta)^{-1}}{V\left(\mathcal{R}^{*}(\delta t)\right)}
$$

where

$$
T=4 \int_{0}^{R} \frac{V(\xi)}{S(\xi)} d \xi
$$

and the function $\mathcal{R}^{*}(\cdot)$ is defined on $(0, T)$ by means of the identity

$$
t=4 \int_{0}^{\mathcal{R}^{*}(t)} \frac{V(\xi)}{S(\xi)} d \xi .
$$

\section{Remarks:}

1. The integrals in (8.15) and (8.16) converge because the integrand is nondecreasing.

2. The number $R$ may be equal to $\infty$ as well. If this is the case then we have by $(8.15)$ $T=\infty$, and the upper bound (8.14) holds for all $t>0$.

3. If $R<\infty$ then both definition (8.16) of $\mathcal{R}^{*}(t)$ and the heat kernel upper bound $(8.14)$ can be extended up to $t=T$.

Examples: In all examples below $R=T=\infty$.

1. Let $V(r)=r^{n}$, then (8.16) gives

$$
\mathcal{R}^{*}(t)=\sqrt{\frac{n}{2} t}
$$


and we get from $(8.14)$

$$
p_{t}(z, z) \leq \frac{\operatorname{const}_{n}}{t^{n / 2}}
$$

By Theorem 7.1 we have also the lower bound

$$
p_{t}(z, z) \geq \frac{\operatorname{const}_{n}}{t^{n / 2}}
$$

Let us note that by Theorem 6.1 we would have only

$$
p_{t}(z, z) \geq \frac{\text { const }}{(t \log t)^{n / 2}} .
$$

2. If $V(r)=e^{k r}$ for large $r$ (and polynomial for small $r$ ) then for large $t$

$$
\mathcal{R}^{*}(t)=\frac{k}{4} t+\text { const }
$$

and

$$
p_{t}(z, z) \leq \frac{\text { const }_{k}}{1-\delta} \exp \left(-\frac{k^{2}}{4} \delta t\right)
$$

Taking $\delta=1-1 / t$, we can write

$$
p_{t}(z, z) \leq \text { const }_{k} t \exp \left(-\frac{k^{2}}{4} t\right)
$$

which gives a sharp exponent $\frac{k^{2}}{4} t$. It is not clear whether the polynomial multiple $t$ in front of the exponential is necessary in general.

3. For $V(r)=e^{r^{\nu}}, \nu \in(0,1)$, we have for large $t$

$$
\mathcal{R}^{*}(t)=\left(\frac{\nu}{4} t+\text { const }\right)^{\frac{1}{2-\nu}}
$$

and taking $\delta=\frac{1}{2}$

$$
p_{t}(z, z) \leq 4 \exp \left(- \text { const }_{\nu} t^{\frac{\nu}{2-\nu}}\right)
$$

To check to what extent (8.17) is sharp, let us apply Theorem 6.1 which yields in this case

$$
\mathcal{R}(t)=t^{\frac{1}{2-\nu}}
$$

and

$$
p_{t}(z, z) \geq \frac{1}{2} \exp \left(-c t^{\frac{\nu}{2-\nu}}\right)
$$

which is in a good agreement with (8.17), at least as far as the exponent $t^{\frac{\nu}{2-\nu}}$ is concerned. Proof of Theorem 8.3. Indeed, let us take $u(x, t)=p_{t}(z, x)$ and try and apply Proposition 8.1. As we know already, any superlevel set $U$ of the function $u(\cdot, t)$ is a ball of some radius $\rho$ centered at the point $z$. By Lemma 8.2, we have

$$
\lambda_{1}(U) \geq \frac{1}{4}\left(\inf _{r \leq \rho} \frac{S(r)}{V(r)}\right)^{2} .
$$


Let us introduce a function $\Lambda(\cdot)$ by the identity

$$
\Lambda(V(\rho))=\frac{1}{4}\left(\inf _{r \leq \rho} \frac{S(r)}{V(r)}\right)^{2}
$$

which is to be true for any $\rho>0$. The function $\Lambda(\cdot)$ is defined on $(0, \infty)$, and is decreasing. Let us note also that for $\rho<R$ we have

$$
\Lambda(V(\rho))=\frac{1}{4}\left(\frac{S(\rho)}{V(\rho)}\right)^{2}
$$

which follows from decreasing of $S(r) / V(r)$ on $(0, R)$.

Since we have for any superlevel set $U$

$$
\lambda_{1}(U) \geq \Lambda(V(\rho))=\Lambda(|U|)
$$

then we can apply Proposition 8.1 and obtain

$$
p_{t}(z, z)=\int_{M} u^{2}(x, t / 2) d x \leq \frac{2(1-\delta)^{-1}}{\gamma(\delta t)}
$$

(we have changed here $\delta$ to $1-\delta$ and $t$ to $t / 2$ ) where $\gamma(\cdot)$ is defined from (8.4):

$$
t=\int_{0}^{\gamma(t)} \frac{d \xi}{\xi \Lambda(\xi)}
$$

Now let us assume that

$$
\gamma(t)<V(R)
$$

(which is a restriction on $t$ ) and change the variable $\xi=V(\rho)$. Since $\xi<V(R)$ then $\rho<R$, and for those $\rho$ the function $\Lambda(V(\rho))$ is defined by (8.18). Hence, we have

$$
t=\int_{0}^{V^{-1}(\gamma(t))} \frac{S(\rho) d \rho}{V(\rho) \Lambda(V(\rho))}=4 \int_{0}^{V^{-1}(\gamma(t))} \frac{V(\rho)}{S(\rho)} d \rho .
$$

By comparing to the definition $(8.16)$ of $\mathcal{R}^{*}(t)$ we see that

$$
V^{-1}(\gamma(t))=\mathcal{R}^{*}(t)
$$

and

$$
\gamma(t)=V\left(\mathcal{R}^{*}(t)\right)
$$

which together with (8.19) yields the upper bound (8.14) .

We are left only to realize that the restriction (8.20) is equivalent to $t<T$. Indeed, let us define $t_{\max }$ from the equation

$$
\gamma\left(t_{\max }\right)=V(R)
$$

which implies by the definition of $\gamma(\cdot)$ that

$$
t_{\max }=\int_{0}^{V(R)} \frac{d \xi}{\xi \Lambda(\xi)}
$$

or, after the same change of variable as above,

$$
t_{\max }=4 \int_{0}^{R} \frac{V(\rho)}{S(\rho)} d \rho=T
$$

what was to be proved. 
8.4 Corollary. Under the conditions of Theorem 8.3, we have for all $t<\min \left(T, R^{2}\right)$

$$
p_{t}(z, z) \leq \frac{2 e(1-\delta)^{-1}}{V(\sqrt{\delta t})}
$$

\section{Remarks:}

1. If $R=\infty$ then (8.21) is valid for all $t>0$.

2. Of course, replacing $\mathcal{R}^{*}(t)$ by $\sqrt{t}$ can make the upper bound not very sharp as occurs for large $t$ for a superpolynomial (or exponential) function $V(r)$. But for a polynomial situation what this statement is aimed at, (8.21) is as sharp as (8.14) .

Proof: It is clear from comparison of (8.14) and (8.21) that it is sufficient to prove

$$
V(\sqrt{t}) \leq e V\left(\mathcal{R}^{*}(t)\right)
$$

To prove $(8.22)$, let us put

$$
g(r)=\frac{V(r)}{S(r)}
$$

and observe that

$$
\int_{r_{1}}^{r_{2}} \frac{d r}{g(r)}=\log \frac{V\left(r_{2}\right)}{V\left(r_{1}\right)}
$$

for any couple $r_{2}>r_{1}>0$. If for a given $t, \mathcal{R}^{*}=\mathcal{R}^{*}(t) \geq \sqrt{t}$ then (8.22) is obvious. Let us suppose $\mathcal{R}^{*}<\sqrt{t}$. Then we have by $(8.23)$

$$
\log \frac{V(\sqrt{t})}{V\left(\mathcal{R}^{*}\right)}=\int_{\mathcal{R}^{*}}^{\sqrt{t}} \frac{d r}{g(r)} \leq \frac{\sqrt{t}-\mathcal{R}^{*}}{g\left(\mathcal{R}^{*}\right)}
$$

where we have used the fact that $g(r)$ is increasing on $(0, R)$ and $\sqrt{t}<R$. On the other hand, we have $\mathcal{R}^{*}(t)<R$ and by the definition of $\mathcal{R}^{*}(t)$

$$
t=4 \int_{0}^{\mathcal{R}^{*}(t)} g(r) d r \leq 4 \mathcal{R}^{*} g\left(\mathcal{R}^{*}\right) \leq\left(\mathcal{R}^{*}+g\left(\mathcal{R}^{*}\right)\right)^{2} .
$$

It implies

$$
\sqrt{t}-\mathcal{R}^{*} \leq g\left(\mathcal{R}^{*}\right)
$$

and by $(8.24)$

$$
\log \frac{V(\sqrt{t})}{V\left(\mathcal{R}^{*}\right)} \leq 1
$$

what was to be proved.

8.5 Corollary. Under the hypotheses of Theorem 8.3, let the function $V(r)$ satisfy in addition the doubling property

$$
V(2 r) \leq A V(r)
$$

for some constant $A$ and for all $r>0$. Then we have for any $t \in(0, T)$

$$
\frac{C_{1}}{V(\sqrt{t})} \leq p_{t}(z, z) \leq \frac{C_{2}}{V(\sqrt{t})}
$$

where $C_{1,2}>0$ depend only on $A$.

Indeed, the upper bound in (8.26) follows from (8.21) because the constant $\delta$ in $V(\sqrt{\delta t})$ can be taken out due to (8.25) . The lower bound (8.26) follows from the upper one and from the doubling volume property by Theorem 7.2 . 


\section{Some examples}

We continue considering the rotationally invariant manifold $M$ constructed in the previous section. In the examples below, we will vary the volume function $V(r)$ although staying within the following limitations:

(a) The total volume $V(\infty)=\infty$.

(b) For some number $r_{0}>0$ and for all $r \in\left(0, r_{0}\right)$ the function $V(r)$ is equal to the Euclidean volume function i.e.

$$
V(r)=v_{n} r^{n}
$$

where $v_{n}$ is the volume of the unit ball in $\mathbb{R}^{n}$. This ensures that the manifold in question has exactly Euclidean metric in the ball $B\left(z, r_{0}\right)$.

(c) For all $r>0$ we would like to have a polynomial upper bound

$$
V(r) \leq C r^{n}
$$

with some constant $C$ and $n=\operatorname{dim} M$.

The purpose of our examples is the following.

$1^{\circ}$ In Theorem 6.1, one cannot make the lower bound (6.5) independent of the geometry of a small neighbourhood of the point $z$. Namely, given only the upper bound (9.1) of the volume function, one cannot get any lower bound for $p_{t}(z, z)$. In fact, we will show that by allowing $r_{0}$ to be arbitrarily small while keeping the same volume upper bound (9.1), one can make $p_{t}(z, z)$ arbitrarily small for a given $t$.

$2^{\circ}$ For the polynomial upper bound (9.1) and with a fixed $r_{0}$, Theorem 6.1 yields for large $t$

$$
p_{t}(z, z) \geq \frac{\text { const }_{r_{0}, n, C}}{(t \log t)^{n / 2}}
$$

We will show that one cannot get rid of the log here. More precisely, we will construct the function $V(r)$ such that for the corresponding heat kernel the quantity

$$
\inf _{t>t_{0}} p_{t}(z, z) t^{n / 2}
$$

is arbitrarily small whereas we have according to (9.2)

$$
\inf _{t>t_{0}} p_{t}(z, z)(t \log t)^{n / 2} \geq \mathrm{const}>0 .
$$

Both examples will eventually come out from the same volume function $V(r)$ which is defined for $r \leq \rho$ as follows:

$$
V(r)=\left\{\begin{array}{c}
C r^{n}, r \leq r_{0} \\
C r_{0}^{n} \exp \left(\eta\left(r-r_{0}\right)\right), r_{0} \leq r \leq \rho
\end{array}\right.
$$

where the numbers $r_{0}<\rho$ are so far arbitrary, $C=v_{n}$, and $\eta$ is chosen to ensure (9.1) for $r=\rho$. Namely, we put

$$
V(\rho)=C \rho^{n}
$$


which gives us the following value for $\eta$

$$
\eta=\frac{n \log \frac{\rho}{r_{0}}}{\rho-r_{0}}
$$

We also need to extend $V(r)$ for $r>\rho$. It can be done in any way with the only requirement that (a) and (c) hold.

It is trivial to check that the function $V(r)$ defined this way does satisfy (a),(b),(c). The function $V(r)$ is virtually smooth except for the point $r=r_{0}$ and, possibly, $r=\rho$ at which it is only continuous. Of course, it can be made smooth by adjusting in a small neighbourhood of the points $r_{0}, \rho$ so that it will not affect all our estimates below. Hence, we will neglect non-smoothness of $V$ and work with it as if it were a smooth function.

Now we would like to apply Theorem 8.3 to get an upper bound for $p_{t}(z, z)$. First of all, let us check that the ratio $\frac{S(r)}{V(r)}$ is non-increasing on $(0, \rho)$. Indeed, we have

$$
\frac{S(r)}{V(r)}=\frac{V^{\prime}(r)}{V(r)}=\left\{\begin{array}{l}
\frac{n}{r}, r<r_{0} \\
\eta, r_{0}<r \leq \rho
\end{array}\right.
$$

where we see from that non-increasing of $\frac{S(r)}{V(r)}$ will follow from

$$
\eta \leq \frac{n}{r_{0}}
$$

In view of (9.3), the inequality (9.4) will follow from

$$
\frac{n \log \frac{\rho}{r_{0}}}{\rho-r_{0}} \leq \frac{n}{r_{0}}
$$

which can be rewritten in the form

$$
\log \frac{\rho}{r_{0}} \leq \frac{\rho}{r_{0}}-1
$$

which is evidently true whenever $\rho>r_{0}$.

In order to apply Theorem 8.3, we have first to calculate $\mathcal{R}^{*}(t)$ from $(8.16)$. Let us define $t_{0}$ and $\tau$ as follows

$$
t_{0}=4 \int_{0}^{r_{0}} \frac{V(r)}{S(r)} d r=\frac{2}{n} r_{0}^{2}, \tau=4 \int_{0}^{\rho} \frac{V(r)}{S(r)} d r .
$$

Then for any $t \in\left(t_{0}, \tau\right]$, the function $\mathcal{R}^{*}(t)$ is determined by

$$
t=4 \int_{0}^{\mathcal{R}^{*}(t)} \frac{V(r)}{S(r)} d r=t_{0}+\int_{r_{0}}^{\mathcal{R}^{*}(t)} \eta^{-1} d r=t_{0}+4 \eta^{-1}\left(\mathcal{R}^{*}(t)-r_{0}\right)
$$

whence we get

$$
\mathcal{R}^{*}(t)=r_{0}+\frac{\eta}{4}\left(t-t_{0}\right)
$$


In particular, we have from (9.6) and $\mathcal{R}^{*}(\tau)=\rho$

$$
\tau=t_{0}+\frac{4}{\eta}\left(\rho-r_{0}\right)
$$

Let us rewrite the upper bound (8.14) in the following way

$$
p_{t}(z, z) \leq \frac{2 t}{(t-s) V\left(\mathcal{R}^{*}(s)\right)}
$$

for any $0<s<t \leq \tau$ (we have just denoted $\delta t$ by $s$ ).

We apply (9.8) for $t=\tau$ and for

$$
s=\tau-\frac{4}{\eta^{2}}
$$

First we have to make sure that $s \in\left(t_{0}, \tau\right)$. To that end, it is sufficient to show that

$$
\tau-t_{0} \geq \frac{4}{\eta^{2}}
$$

This will follow from

$$
\frac{\eta}{4}\left(\tau-t_{0}\right) \geq \frac{1}{\eta}
$$

which is by (9.7) the same as

$$
\rho-r_{0} \geq \frac{1}{\eta}
$$

or, by (9.3) ,

$$
n \log \frac{\rho}{r_{0}} \geq 1
$$

This is true if we assume for all our further considerations that $\rho \geq e r_{0}$.

Therefore, we have for the chosen $t$ and $s$ by $(9.8),(9.6),(9.9),(9.7)$, and (9.3)

$$
\begin{aligned}
p_{\tau}(z, z) & \leq \frac{2 \tau}{(\tau-s) C r_{0}^{n}} \exp \left(-\eta\left(\mathcal{R}^{*}(s)-r_{0}\right)\right) \\
& =\frac{2 \tau}{(\tau-s) C r_{0}^{n}} \exp \left(-\frac{\eta^{2}}{4}\left(s-t_{0}\right)\right) \\
& =\frac{\eta^{2} \tau}{2 C r_{0}^{n}} \exp \left(-\frac{\eta^{2}}{4}\left(\left(\rho-r_{0}\right) \frac{4}{\eta}-\frac{4}{\eta^{2}}\right)\right) \\
& =\frac{\eta^{2} \tau}{2 C r_{0}^{n}} \exp \left(-n \log \frac{\rho}{r_{0}}+1\right) \\
& =\frac{e \eta^{2} \tau}{2 C \rho^{n}} .
\end{aligned}
$$

Thus, we obtain

$$
p_{\tau}(z, z) \leq \frac{e \eta^{2} \tau}{2 C \rho^{n}}
$$


We consider $r_{0}, \rho$ as parameters to be chosen. All other parameters $\eta, t_{0}, \tau$ are determined by (9.3), (9.5), and (9.7) respectively. The two examples below differ by the choice of $r_{0}, \rho$. In the first example, we take $\rho$ to be large enough (eventually we let $\rho \rightarrow \infty$ ) while $r_{0}$ is defined by

$$
r_{0}=\exp \left(-\frac{\rho^{2}}{n}\right)
$$

and, therefore, $r_{0}$ will go to 0 .

We have by (9.3) and (9.11) the lower and upper bounds for $\eta$ :

$$
\eta \geq \frac{n \log \frac{1}{r_{0}}}{\rho}=\rho
$$

and

$$
\eta=\frac{n \log \rho+n \log \frac{1}{r_{0}}}{\rho-r_{0}}=\frac{n \log \rho+\rho^{2}}{\rho-r_{0}} \leq 2 \rho
$$

for large enough $\rho$. Let us estimate also $\tau$ from (9.7) and (9.12) :

$$
\tau=t_{0}+\frac{4\left(\rho-r_{0}\right)}{\eta} \leq \frac{2}{n} r_{0}^{2}+4-\frac{4 r_{0}}{\rho}
$$

which implies

$$
\tau<4
$$

for large enough $\rho$ (because of (9.11) ).

Finally, we use the fact that $p_{t}(z, z)$ is decreasing in $t$ and the upper bounds (9.13) and (9.14) of $\eta$ and $\tau$ respectively to get from (9.10)

$$
p_{4}(z, z) \leq p_{\tau}(z, z) \leq \frac{8 e}{C} \rho^{2-n}
$$

whence we see that for large enough $\rho$ (and for $n>2$ ) the value of $p_{4}(z, z)$ may be arbitrarily small whereas we have the uniform upper bound (9.1) for the volume $V(r)$ which remains the same regardless of whatever $\rho, r_{0}$ are chosen.

In the second example, we keep $r_{0}>0$ fixed while $\rho$ will be again large. We have from $(9.10)$

$$
\tau^{\frac{n}{2}} p_{\tau}(z, z) \leq \frac{e \eta^{2} \tau^{\frac{n}{2}+1}}{2 C \rho^{n}}=\frac{e}{2 C} \frac{\eta^{2}}{\rho^{n}}\left(t_{0}+\frac{4}{\eta}\left(\rho-r_{0}\right)\right)^{\frac{n}{2}+1}
$$

Now we let $\rho \rightarrow \infty$ while $r_{0}$ stays constant. Then $t_{0}$ does not change whereas $\eta \rightarrow 0$ because as follows from (9.3)

$$
\eta \sim n \frac{\log \rho}{\rho}
$$

as $\rho \rightarrow \infty$. Therefore, we get for large enough $\rho$

$$
\tau^{\frac{n}{2}} p_{\tau}(z, z) \leq \operatorname{const} \frac{\eta^{2}}{\rho^{n}}\left(\frac{\rho}{\eta}\right)^{\frac{n}{2}+1}=\operatorname{const}(\rho \eta)^{-\frac{n}{2}+1},
$$


and

$$
\tau^{\frac{n}{2}} p_{\tau}(z, z) \leq \operatorname{const}(\log \rho)^{-\frac{n}{2}+1}
$$

which can be made arbitrarily small provided $\rho$ is large enough and $n>2$.

Thus, the quantity

$$
t^{\frac{n}{2}} p_{t}(z, z)
$$

does not have a lower bound while by Theorem 6.1 the quantity

$$
(t \log t)^{\frac{n}{2}} p_{t}(z, z)
$$

is bounded from below by a positive constant.

\section{Remarks:}

1. Let us notice also that the same example shows that for any $\varepsilon>0$ the quantity

$$
t^{\frac{n}{2}} \log ^{\frac{n}{2}-1-\varepsilon} t p_{t}(z, z)
$$

does not have any lower bound either. Indeed, as follows from (9.7)

$$
\log \tau \sim \log \rho
$$

as $\rho \rightarrow \infty$. Therefore, we get from (9.15)

$$
\tau^{\frac{n}{2}} \log ^{\frac{n}{2}-1-\varepsilon} \tau p_{\tau}(z, z) \leq \operatorname{const}(\log \rho)^{-\varepsilon} \rightarrow 0
$$

as $\rho \rightarrow \infty$.

2. Since $\eta$ stays bounded as $\rho$ goes to zero, the family of manifolds built above has uniformly bounded geometry. This indicates that by discretisation, one should be able to draw the same conclusion for random walks on graphs. In other terms, Theorem 3, (ii) in $[\mathrm{L}]$ is likely to be optimal.

\section{Lower bounds and volume growth}

Let us suppose that for some point $z \in M$ and for some numbers $0<r_{0}<r_{1}<r_{2} \leq \infty$ we have for any $r \in\left(r_{1}, r_{2}\right)$

$$
V(z, r) \leq v(r)
$$

and

$$
V\left(z, r_{0}\right) \geq v_{0}
$$

where $v(\cdot)$ is a continuous increasing function on $\left[r_{1}, r_{2}\right)$, and $v_{0}>0$. Under these conditions, we would like to have a lower bound for $\sup _{x} p_{t}(x, x)$. To introduce it, we define a function $\mathcal{R}(t)$ similar to that of $\S 6$. Namely, let us assume in addition that $v\left(r_{1}\right)>2$, and the function

$$
\frac{r^{2}}{\log v(r)}
$$

is strictly increasing on $\left(r_{1}, r_{2}\right)$. Therefore, it has an inverse function which will be denoted by $\mathcal{R}(t)$. Let the domain of $\mathcal{R}(t)$ be the interval $\left(t_{1}, t_{2}\right)$. 
10.1 Theorem. Let the manifold $M$ be stochastically complete. Then under the above assumptions, we have for any $t \in\left(t_{1}, t_{2}\right)$

$$
\sup _{x} p_{t}(x, x) \geq \frac{0.5}{V(z, \mathcal{R}(a t))}
$$

where $a=a\left(r_{0}, r_{1}, v_{0}\right)$.

\section{Remarks:}

1. Let us notice the following distinctions of this theorem from Theorem 6.1. While the latter provides a stronger conclusion - a pointwise lower bound rather than sup - lower bound, the former requires less in the hypotheses. Indeed, instead of a Faber-Krahn inequality in the ball $B\left(z, r_{0}\right)$, we now need to know only a lower bound of the volume $V\left(z, r_{0}\right)$. Then, the upper bound of the volume function must not necessarily be true up to $\infty$, or, in other words, $r_{2}$ may be finite as well as infinite. Thus, Theorem 10.1 applies also for a certain bounded range of radii. The question whether it is possible to drop the condition (10.2) remains open.

2. If $r_{2}=\infty$ then the hypothesis of stochastic completeness is superfluous because it follows from increasing of the function (10.3) in the same way as in Theorem 6.1.

3. Let us mention also that a particular case of this theorem for a function

$$
v(r)=\exp \left(r^{\alpha}\right), \alpha \in(0,1]
$$

was proved by F.Lust-Piquard [L] by a different method.

Proof of the theorem. The main tool in the proof is the inequality proved first by Takeda $[\mathrm{T}]$ which provides an upper bound of the double integral of the heat kernel taken over a direct product of a ball and the exterior of its enlargement. There are different versions of that inequality: the original one by $[\mathrm{T}]$, a numerically improved one by T.Lyons [Ly], and one obtained by purely analytic methods in [G3]. For technical reasons, we will use the third version. It says that on an arbitrary complete manifold $M$, for any point $z \in M$, and for any radii $0<r<R$ the following inequality holds

$$
\int_{B(z, r)} \int_{M \backslash B(z, R)} p_{t}(x, y) d x d y \leq \sqrt{V(z, r) V(z, R)} F\left(\frac{R-r}{\sqrt{2 t}}\right)
$$

where

$$
F(\xi)=\left(\xi+\frac{1}{\xi}\right) \exp \left(-\frac{\xi^{2}}{2}+\frac{1}{2}\right)
$$

(different versions of Takeda's inequality have different functions $F$ ).

The expression for $F(\xi)$ can be slightly simplified for $\xi \geq 1$ which is the only case we shall need. Indeed, one can check easily that for such $\xi$ we have the inequality

$$
\xi+\frac{1}{\xi} \leq \exp \left(\frac{\xi^{2}}{4}+\frac{1}{2}\right)
$$

which yields

$$
F(\xi) \leq \exp \left(-\frac{\xi^{2}}{4}+1\right)
$$


whenever $\xi \geq 1$.

The inequality (10.5) implies obviously that for some $y \in B(z, r)$

$$
\int_{M \backslash B(z, R)} p_{t}(x, y) d x \leq \sqrt{\frac{V(z, R)}{V(z, r)}} F\left(\frac{R-r}{\sqrt{2 t}}\right)
$$

or, using the fact that the manifold is stochastically complete,

$$
\int_{B(z, R)} p_{t}(x, y) d x \geq 1-\sqrt{\frac{V(z, R)}{V(z, r)}} F\left(\frac{R-r}{\sqrt{2 t}}\right) .
$$

Therefore, for some $x \in B(z, R)$

$$
p_{t}(x, y) \geq \frac{1-\sqrt{\frac{V(z, R)}{V(z, r)}} F\left(\frac{R-r}{\sqrt{2 t}}\right)}{V(z, R)} .
$$

Since by the semigroup property $p_{t}(x, y) \leq \sqrt{p_{t}(x, x) p_{t}(y, y)}$ then (10.8) implies

$$
\sup _{x \in B(z, R)} p_{t}(x, x) \geq \frac{1-\sqrt{\frac{V(z, R)}{V(z, r)}} F\left(\frac{R-r}{\sqrt{2 t}}\right)}{V(z, R)} .
$$

Let us now fix some $t \in\left(t_{1}, t_{2}\right)$, put $r=r_{0}$ and optimize (or nearly optimize) the inequality (10.9) with respect to $R \in\left(r_{1}, r_{2}\right)$. We can rewrite (10.9) as follows

$$
\sup _{x} p_{t}(x, x) \geq \frac{1-\sqrt{\frac{v(R)}{v_{0}}} F\left(\frac{R-r_{0}}{\sqrt{2 t}}\right)}{V(z, R)} .
$$

It will imply

$$
\sup _{x} p_{t}(x, x) \geq \frac{0.5}{V(z, R)}
$$

provided $R$ is chosen so that

$$
\sqrt{\frac{v(R)}{v_{0}}} F\left(\frac{R-r_{0}}{\sqrt{2 t}}\right) \leq \frac{1}{2} .
$$

This inequality follows in turn from the next one which is obtained by applying the upper bound (10.7) for the function $F$

$$
\frac{v(R)}{v_{0}} \exp \left(-\frac{\left(R-r_{0}\right)^{2}}{4 t}+2\right) \leq \frac{1}{4}
$$

provided $R$ is so that

$$
\frac{R-r_{0}}{\sqrt{2 t}} \geq 1
$$


Thus, we will have the heat kernel lower bound (10.11) whenever we have chosen $R \in$ $\left(r_{1}, r_{2}\right)$ satisfying $(10.13)$ and $(10.12)$.

We claim that this is true for $R=\mathcal{R}(a t)$ with a suitable constant $a$. Let us check first (10.13) . We have by the definition of the function $\mathcal{R}$

$$
a t=\frac{R^{2}}{\log v(R)}
$$

Therefore,

$$
\begin{aligned}
\frac{\left(R-r_{0}\right)^{2}}{2 t} & =\left(1-\frac{r_{0}}{R}\right)^{2} \frac{R^{2}}{2 t} \\
& \geq\left(1-\frac{r_{0}}{r_{1}}\right)^{2} \frac{a}{2} \log v(R) \\
& \geq\left(1-\frac{r_{0}}{r_{1}}\right)^{2} \frac{a}{2} \log 2
\end{aligned}
$$

(where we have used $v(R) \geq v\left(r_{1}\right) \geq 2$ ) whence (10.13) follows provided

$$
a \geq \frac{2}{\left(1-\frac{r_{0}}{r_{1}}\right)^{2} \log 2} .
$$

To check (10.12), let us transform it to the form

$$
4 t \leq \frac{\left(R-r_{0}\right)^{2}}{\log \frac{v(R)}{v_{0}}+4}
$$

(where we have used $2+\log 4<4$ ). Taking into account $(10.14$ ), this will follow from

$$
\frac{4}{a} \frac{R^{2}}{\log v(R)} \leq \frac{\left(R-r_{0}\right)^{2}}{\log \frac{v(R)}{v_{0}}+4}
$$

or from

$$
a \geq\left(\frac{2 R}{R-r_{0}}\right)^{2} \frac{\log \frac{v(R)}{v_{0}}+4}{\log v(R)} .
$$

This inequality will, in turn, follow from

$$
a \geq\left(\frac{2}{1-\frac{r_{0}}{R}}\right)^{2}\left(1+\frac{4-\log v_{0}}{\log v(R)}\right) .
$$

If $\log v_{0} \geq 4$ then this inequality follows from

$$
a \geq\left(\frac{2}{1-\frac{r_{0}}{r_{1}}}\right)^{2}
$$

otherwise it follows from

$$
a \geq\left(\frac{2}{1-\frac{r_{0}}{r_{1}}}\right)^{2}\left(1+\frac{4-\log v_{0}}{\log 2}\right)
$$


where we have used that $v(R) \geq v\left(r_{1}\right) \geq 2$.

Let us take $a=a\left(r_{0}, r_{1}, v_{0}\right)$ to satisfy all $(10.15),(10.16),(10.17)$. For this $a$ and for $R=\mathcal{R}(a t)$, we have (10.13) and (10.12) and, thereby, the lower bound (10.11) what was to be proved.

Remark: The basic inequality (10.9) is applicable also under different assumptions about the manifold. For example, let us assume that the volume function $V(z, r)$ satisfies the doubling volume condition. Then by taking $R=\operatorname{const} \sqrt{t}$ and $r=\frac{1}{2} R$ one obtains for suitable constants

$$
\sup _{x} p_{t}(x, x) \geq \frac{\text { const }}{v(\sqrt{t})}
$$

which coincides with Theorem 2.7.

Acknowledgment: We would like to thank Christophe Pittet who helped us to work out Propositions 4.9 and 4.10 .

\section{REFERENCES}

[Al] Alexopoulos G., A lower estimate for central probabilities on polycyclic groups, Can. J. Math., 44 no.5, (1992) 897 - 910.

[Ar] Aronson D.G., Non-negative solutions of linear parabolic equations, Ann. Scuola Norm. Sup. Pisa. Cl. Sci. (4), 22 (1968) 607 - 694. Addendum 25 (1971) 221 - 228.

[Az] Azencott R., Behavior of diffusion semi-groups at infinity, Bull. Soc. Math. (France), 102 (1974) $193-240$.

[BCF] Benjamini I., Chavel I., Feldman E.A., Heat kernel lower bounds on Riemannian manifolds using the old ideas of Nash, Proc. London Math. Soc., 72 (1996) 215 - 240.

[CCMP] Carey A., Coulhon T., Mathai V., Phillips J., Von Neumann spectra near the mass gap, Bull. Sci. Math., to appear.

[CKS] Carlen E.A., Kusuoka S., Stroock D.W., Upper Bounds for symmetric Markov transition functions, Ann. Inst. H. Poincaré, proba. et stat., suppl. au no.2, (1987) $245-287$.

[Carn] Carne K., A transmutation formula for Markov chains, Bull. Sc. Math., 2eme serie, 109 (1985) $399-403$.

[Carr] Carron G., Inégalités isopérimétriques de Faber-Krahn et conséquences, in: "Actes de la table ronde de géométrie différentielle en l'honneur de Marcel Berger", Collection SMF Séminaires et congrès, no.1, 1994.

[Ch] Cheeger J., A lower bound for the smallest eigenvalue of the Laplacian, in: Problems in Analysis: A Symposium in honor of Salomon Bochner, Princeton University Press. Princeton, 1970. $195-199$.

[ChY] Cheeger J., Yau S.-T., A lower bound for the heat kernel, Comm. Pure Appl. Math., 34 (1981) $465-480$.

[C1] Coulhon T., Inégalités de Gagliardo-Nirenberg pour les semi-groupes d'opérateurs et applications, Potential Anal., 1 (1992) $343-353$.

[C2] Coulhon T., Ultracontractivity and Nash type inequalities, J. Funct. Anal., 141 (1996) $510-539$.

[C3] Coulhon T., Dimensions at infinity for Riemannian manifolds, Potential Anal., 4 (1995) no.5, $335-344$. 
[CL] Coulhon T., Ledoux M., Isopérimétrie, décroissance du noyau de la chaleur et transformations de Riesz: un contre-exemple, Ark. Mat., 32 (1994) 63 - 77.

[CS1] Coulhon T., Saloff-Coste L., Isopérimétrie pour les groupes et les variétés, Revista Mathemática Iberoamericana, 9 no.2, (1993) 293 - 314.

[CS2] Coulhon T., Saloff-Coste L., Minorations pour les chaînes de Markov unidimensionnelles, Probab. Theory Relat. Fields, 97 (1993) $423-431$.

[D1] Davies E.B., "Heat kernels and spectral theory", Cambridge: Cambridge University Press, 1989.

[D2] Davies E.B., Non-Gaussian aspects of heat kernel behaviour, J. London Math. Soc., to appear.

[FS] Fabes E.B., Stroock D.W., A new proof of Moser's parabolic Harnack inequality via the old ideas of Nash, Arch. Rat. Mech. Anal., 96 (1986) 327 - 338.

[G1] Grigor'yan A.A., On stochastically complete manifolds, (in Russian) DAN SSSR, 260 (1986) no.3, 534-537. Engl. transl. Soviet Math. Dokl., 34 (1987) no.2, 310-313.

[G2] Grigor'yan A., Heat kernel upper bounds on a complete non-compact manifold, Revista Mathemática Iberoamericana, 10 no.2, (1994) 395 - 452.

[G3] Grigor'yan A., Integral maximum principle and its applications, Proc. of Edinburgh Royal Society, 124A (1994) $353-362$.

[G4] Grigor'yan A., Heat kernel on a non-compact Riemannian manifold, in: "Proceedings of Symposia in Pure Mathematics, 1993 Summer Research Institute on Stochastic Analysis", ed. M.Pinsky, 1994.

[G5] Grigor'yan A.A., Stochastically complete manifolds and summable harmonic functions, (in Russian) Izv. AN SSSR, ser. matem., 52 no.5, (1988) 1102 - 1108 . Engl. transl. Math. USSR Izvestiya, 33 no.2, (1989) 425 - 432.

[G6] Grigor'yan A.A., The heat equation on non-compact Riemannian manifolds, (in Russian) Matem. Sbornik, 182 (1991) no.1, 55 - 87. Engl. transl. Math. USSR Sb., 72 (1992) no.1, $47-77$.

[G7] Grigor'yan A., Gaussian upper bounds for the heat kernel and for its derivatives on a Riemannian manifold, in: "Proceeding of the ARW on Potential Theory, Chateau de Bonas, July 1993", ed. K.GowriSankaran, Kluwer Academic Publisher, 1994. $237-252$.

[Gu] Guivarc'h Y., Sur la loi des grands nombres et le rayon spectral d'une marche aléatoire, Journée sur les marches aléatoires, Astérisque, 74 (1980) 47 - 98.

[HS] Hoffman D., Spruck J., Sobolev and isoperimetric inequalities for Riemannian submanifolds, Comm. Pure Appl. Math., 27 (1974) 715 - 725.

[KL] Karp L., Li P., The heat equation on complete Riemannian manifolds, unpublished.

[La] Lalley S. P., Finite range random walk on free groups and homogeneous trees, Ann. Prob., 21 (1993) no.4, 2087 - 2130.

[LY] Li P., Yau S.-T., On the parabolic kernel of the Schrödinger operator, Acta Math., 156 (1986) no.3-4, $153-201$.

[L] Lust-Piquard F., Lower bounds on $\left\|K^{n}\right\|_{1 \rightarrow \infty}$ for some contraction $K$ of $L^{2}(\mu)$, with some applications to Markov operators, Math.Ann., 303 (1995) 699 - 712.

[Ly] Lyons T., Random thoughts on reversible potential theory, in: Summer School in Potential Theory, Joensuu 1990, edited by Ilpo Laine, University of Joensuu. Publications in Sciences 26, ISBN 951-696-837-6., 71 - 114.

[N] Nash J., Continuity of solutions of parabolic and elliptic equations, Amer. J. Math., 80 (1958) $931-954$. 
[P] Pittet C., Folner sequences on polycyclic groups, Revista Mathemática Iberoamericana, 11 no.3, (1995) $675-685$.

[PE] Porper F.O., Eidel'man S.D., Two-side estimates of fundamental solutions of second-order parabolic equations and some applications, (in Russian) Uspechi Mat. Nauk, 39 (1984) no.3, 101 - 156. Engl. transl. Russian Math. Surveys, 39 (1984) no.3, $119-178$.

[S] Saloff-Coste L., A note on Poincaré, Sobolev, and Harnack inequalities, Duke Math J., I.M.R.N., 2 (1992) $27-38$.

[St] Stroock D.W., Estimates on the heat kernel for the second order divergence form operators, in: "Probability theory", Proceedings of the 1989 Singapore Probability Conference held at the National University of Singapore, June 8-16, 1989, L.H.Y Chen, K. Choi, K. Hu and J.H. Lou, eds., 1992. De Gruyter, $29-44$.

[Stu] Sturm K-Th., Analysis on local Dirichlet spaces I, Recurrence, conservativeness and $L^{p}$-Liouville properties, J. Reine. Angew. Math., 456 (1994) $173-196$.

[T] Takeda M., On a martingale method for symmetric diffusion process and its applications, Osaka J. Math, 26 (1989) 605 - 623.

[V1] Varopoulos N.Th., Long range estimates for Markov chains, Bull. Sc.Math., 2eme serie, 109 (1985) $113-119$.

[V2] Varopoulos N.Th., Hardy-Littlewood theory for semigroups, J. Funct. Anal., 63 (1985) no.2, $240-260$.

[V3] Varopoulos N.Th., Small time Gaussian estimates of heat diffusion kernel.I. The semigroup technique, Bull. Sci. Math.(2), 113 (1989) no.3, 253 - 277. 